\title{
Modelagem Bidimensional de Transporte de Escalares Não-Conservativos em Corpos d'Água Rasos
}

\author{
Cynara L. da Nóbrega Cunha, Teófilo C. N. Monteiro \\ Depto. de Saneamento e Saúde Ambiental - ENSP - FIOCRUZ - Rua Leopoldo Bulhões, 1480/50 andar \\ 21041-210 Rio de Janeiro, RJ - cynaracarrer@uol.com.br, teofilo@ensp.fiocruz.br \\ Paulo C. C. Rosman \\ Programa de Engenharia Oceânica - Área de Engenharia Costeira - COPPE/UFRJ - Caixa Postal 68508 \\ 21945-970 Rio de Janeiro, RJ-pccr@peno.coppe.ufrj.br
}

Recebido: 16/05/01 - revisão: 17/09/01 - aceito: 19/04/02

\begin{abstract}
RESUMO
Neste trabalho é desenvolvido um modelo bidimensional de transporte, aplicado a escalares passivos e nãoconservativos. O modelo usa a equação de transporte integrada na direção vertical, elementos finitos na discretização espacial e diferenças finitas na discretização temporal. A parametrização das tensões turbulentas é baseada nas técnicas de filtragem, que são derivadas da técnica conhecida como Simulação dos Grandes Vórtices (LES, Large Eddy Simulation). A validação do modelo é demonstrada através da solução numérica de experimentos que possuem soluções analíticas conhecidas, considerando diversos tipos de fontes. O modelo foi aplicado ainda na simulação do transporte de sal na Baía de Sepetiba, mostrando a sua aplicabilidade em estuários com batimetria e geometria complexas.
\end{abstract}

Palavras-chave: modelagem bidimensional; transporte de escalares.

\section{INTRODUÇÃO}

As regiões costeiras vêm se transformando em importantes áreas, não só no ponto de vista econômico mas, principalmente, no ponto de vista ambiental, tendo sofrido ao longo das últimas décadas uma série de processos de degradação. Os principais processos de degradação estão ligados ao lançamento da carga de escalares nas regiões costeiras, quer de forma pontual ou difusa. Diante deste quadro, surge a necessidade de se criar programas de gerenciamento ambiental, estabelecendo padrões bem definidos de utilização e exploração destas áreas.

Dentro deste contexto, foi desenvolvido um Modelo de Transporte Euleriano Advectivo-Difusivo integrado na vertical, para escalares passivos e nãoconservativos. Neste tipo de modelo, o campo de velocidades é conhecido, ou seja, o escalar transportado não altera a hidrodinâmica do corpo d'água receptor. Como conseqüência, a modelagem do padrão hidrodinâmico do corpo d'água e a modelagem do transporte do escalar são problemas desacoplados. Assim, para que ocorra uma boa simulação do transporte do escalar, uma adequada simulação do campo hidrodinâmico torna-se necessário.
Os escalares não-conservativos, que representam a maioria das substâncias existentes na água, sofrem modificação de concentração através de processos físicos, biológicos e químicos. $\mathrm{O}$ entendimento e a formulação destes processos são, portanto, fundamentais na construção do modelo de transporte.

Considerando a natureza e as possíveis fontes dos escalares de interesse, torna-se evidente que uma vasta gama de escalas de problemas pode surgir. Se, por exemplo, quer-se investigar o transporte de escalar ao longo de um corpo d'água, como o transporte de sal em uma baía, a escala de interesse deverá possivelmente compreender toda a área do corpo d'água, e ser resolvida a partir de modelos de transporte do tipo euleriano. No entanto, se o interesse reside na pluma de um emissário de esgoto, as escalas de interesse deverão ser da ordem de poucos quilômetros e serão bem resolvidas através de modelo de transporte do tipo lagrangeano.

Os modelo eulerianos resolvem a equação de transporte sobre um malha fixa, diferentemente dos modelos lagrangeanos, onde a malha se move junto com o escalar. Os modelos tipo euleriano têm dificuldade em descrever o transporte de escalares, quando a dimensão da fonte é pequena comparada com a discretização espacial empregada. No caso 
de emissários, este tipo de modelo só deve ser empregado quando a malha tiver dimensões compatíveis com a dimensão do difusor, o que torna o custo computacional bastante elevado na simulação deste tipo de problema.

A integração na direção vertical sugere que este modelo deva ser aplicado em corpos d'água rasos, que possuem dimensões horizontais preponderantes sobre a dimensão vertical, com a coluna d'água bem misturada, onde a estratificação vertical é pouco relevante. Neste tipo de corpo receptor, o campo de velocidades usado é médio na vertical, não permitindo a descrição do perfil de velocidades.

O transporte de uma dada substância em um corpo d'água é dominado pela advecção, sugerindo assim uma enorme dependência entre a simulação hidrodinâmica e o processo de transporte. No entanto, os processos difusivos também são muito importantes para a definição do transporte. No modelo desenvolvido, a parte difusiva é modelada segundo as técnicas de filtragem (Aldama,1985 e Rosman,1987), que são derivadas da técnica conhecida como Simulação de Grandes Vórtices (LES, Large Eddy Simulation).

Neste artigo é mostrado um Modelo de Transporte Euleriano Advectivo-Difusivo, chamado FIST-ADE, integrado na vertical, para escalares passivos e não-conservativos, com reações cinéticas de primeira ordem. Este modelo foi desenvolvido para compor o Sistema de Base Hidrodinâmica Ambiental, chamado SisBAHIA, desenvolvido pela Área de Engenharia Costeira e Oceanográfica do Programa de Engenharia Oceânica da COPPE/UFRJ. O SisBAHIA é capaz de realizar modelagem ambiental de corpos d'água costeiros, sendo constituído por um modelo de circulação hidrodinâmica tridimensional para corpos d'água rasos, um modelo de transporte Euleriano Advectivo-Difusivo, o FIST-ADE, e um modelo de transporte Lagrangeano Advectivo-Difusivo. Maiores informações sobre o SisBAHIA podem ser obtidas em Rosman (2000).

A validação do código computacional foi feita comparando os resultados numéricos, obtidos pelo FIST-ADE, com soluções analíticas, desenvolvidos para uma fonte instantânea e para uma fonte contínua, considerando apenas os processos difusivos e depois incluindo a advecção, obtendo-se uma boa aproximação entre as duas soluções.

Com o objetivo de mostrar a potencialidade frente à modelagem ambiental de corpos d'água rasos, o FIST-ADE foi aplicado à Baía de Sepetiba, simulando o transporte de sal. Vale lembrar que o objetivo desta aplicação não é mostrar qualquer tipo de calibração em relação aos valores medidos, mas sim mostrar a potencialidade do FIST-ADE na simulação do transporte de sal em um corpo d'água com geometria complexa e avaliar o seu desempenho.

\section{MODELO MATEMÁTICO}

A equação bidimensional que descreve o transporte de um escalar para variáveis médias ou de grande escala (Bedford, 1994), usando a técnica de filtragem para a modelagem das tensões turbulentas (mais detalhes em Rosman, 1987 e Aldama, 1985), é dada por:

$$
\left.\frac{\partial \mathrm{C}}{\partial \mathrm{t}}+\mathrm{U}_{\mathrm{i}} \frac{\partial \mathrm{C}}{\partial \mathrm{x}_{\mathrm{i}}}=\frac{1}{\mathrm{H}} \frac{\partial}{\partial \mathrm{x}_{\mathrm{j}}}\left(\mathrm{H}\left[\mathrm{D}_{\mathrm{ij}} \delta_{\mathrm{jk}}+\frac{\Lambda_{\mathrm{k}}^{2}}{12} \mid \frac{\partial \mathrm{U}_{\mathrm{j}}}{\partial \mathrm{x}_{\mathrm{k}}}\right]\right] \frac{\partial \mathrm{C}}{\partial \mathrm{x}_{\mathrm{k}}}\right)+\sum \mathrm{R}
$$

onde $\mathrm{C}$ é a concentração do escalar de interesse, $\mathrm{U}_{\mathrm{i}}$ são as componentes da velocidade na direção $x_{i}$ promediadas na direção vertical, $\Sigma \mathrm{R}$ representa as fontes ou as perdas de massa integrada na direção vertical, $\mathrm{H}$ é a altura da coluna d'água, $\mathrm{D}_{\mathrm{ij}}$ é o tensor que representa o coeficiente de difusão turbulenta de massa, $\delta_{\mathrm{jk}}$ representa o delta de Kronecker e $\Lambda_{\mathrm{k}}=\alpha_{\mathrm{k}} \Delta \mathrm{x}_{\mathrm{k}}$ é a largura do filtro na dimensão $\mathrm{x}_{\mathrm{k}}$ sendo $\alpha_{\mathrm{k}}$ um parâmetro de escala. Os valores típicos de $\alpha_{k}$ calibrados para difusão/advecção são da ordem de 0.25 a 2.0, com valor usual igual a 1.0. Na Equação (1), i, j= 1, 2 e k=1, 2, 3, sendo $k=3$ correspondente ao tempo $t\left(\right.$ no contexto $\left.x_{3}=t\right)$.

O coeficiente $D_{i j}$ representa a mistura devida à turbulência gerada principalmente pelo fundo, podendo ser decomposto segundo as direções longitudinal e transversal:

$$
\begin{aligned}
& D_{x x}=D_{L} \cos ^{2} \varphi+D_{T} \sin ^{2} \varphi \\
& D_{x y}=D_{y x}=\left(D_{L}-D_{T}\right) \sin \varphi \cos \varphi \\
& D_{y y}=D_{L} \sin ^{2} \varphi+D_{T} \cos ^{2} \varphi
\end{aligned}
$$

onde $\mathrm{D}_{\mathrm{L}}$ e $\mathrm{D}_{T}$ são, respectivamente, os coeficientes nas direções longitudinal e transversal e $\varphi$ é o ângulo entre a linha de corrente e o eixo $x$. O coeficiente de mistura turbulenta transversal é 40 vezes menor que o longitudinal devido, principalmente, ao fato de que os gradientes de mistura transversais são muito menores que os gradientes longitudinais. Fischer (1979), parametrizou estes coeficientes como segue:

$$
\begin{aligned}
& \mathrm{D}_{\mathrm{L}}=\alpha\left(5.93\left|\mathrm{u}_{*}\right| \mathrm{H}\right) \\
& \mathrm{D}_{\mathrm{T}}=\beta\left(0.15\left|\mathrm{u}_{*}\right| \mathrm{H}\right)
\end{aligned}
$$


onde $\alpha$ e $\beta$ são parâmetros incluídos para facilitar a calibração e $\mathrm{u}_{*}$ é a velocidade de atrito característica.

Voltando à Equação (1) e expandindo as derivadas (Rosman, 2000), obtém-se:

$$
\begin{aligned}
& \left(1-u_{x t}-v_{y t}\right) \frac{\partial C}{\partial t}+\left(U-u_{x x}-v_{y x}\right) \frac{\partial C}{\partial x}+ \\
& \left(V-u_{x y}-v_{y y}\right) \frac{\partial C}{\partial y}=\frac{T_{x x}}{H} \frac{\partial C^{2}}{\partial x^{2}}+\frac{T_{x y}}{H} \frac{\partial}{\partial x} \\
& \left(\frac{\partial C}{\partial y}\right)+\frac{T_{x t}}{H} \frac{\partial}{\partial x}\left(\frac{\partial C}{\partial t}\right)+\frac{T_{y x}}{H} \frac{\partial}{\partial y}\left(\frac{\partial C}{\partial x}\right)+ \\
& \frac{T_{y y}}{H} \frac{\partial C^{2}}{\partial y^{2}}+\frac{T_{y t}}{H} \frac{\partial}{\partial y}\left(\frac{\partial C}{\partial t}\right)+\sum R
\end{aligned}
$$

onde $\mathrm{U}$ e $\mathrm{V}$ são as componentes da velocidade na direção x e y, respectivamente, e:

$$
\begin{aligned}
& \mathrm{T}_{\mathrm{xx}}=\mathrm{H}\left[\mathrm{D}_{\mathrm{xx}}+\frac{\Lambda_{\mathrm{x}}^{2}}{12}\left|\frac{\partial \mathrm{U}}{\partial \mathrm{x}}\right|\right] \\
& T_{x y}=H\left[D_{x y}+\frac{\Lambda_{y}^{2}}{12}\left|\frac{\partial U}{\partial y}\right|\right] \\
& \mathrm{T}_{\mathrm{xt}}=\mathrm{H} \frac{\Lambda_{\mathrm{t}}^{2}}{12}\left|\frac{\partial \mathrm{U}}{\partial \mathrm{t}}\right| \\
& \mathrm{T}_{\mathrm{yx}}=\mathrm{H}\left[\mathrm{D}_{\mathrm{yx}}+\frac{\Lambda_{\mathrm{x}}^{2}}{12}\left|\frac{\partial \mathrm{V}}{\partial \mathrm{x}}\right|\right] \\
& \mathrm{T}_{\mathrm{yy}}=\left(\mathrm{H}\left[\mathrm{D}_{\mathrm{yy}}+\frac{\Lambda_{\mathrm{y}}^{2}}{12}\left|\frac{\partial \mathrm{V}}{\partial \mathrm{y}}\right|\right]\right) \\
& \mathrm{T}_{\mathrm{yt}}=\mathrm{H} \frac{\Lambda_{\mathrm{t}}^{2}}{12}\left|\frac{\partial \mathrm{V}}{\partial \mathrm{t}}\right| \\
& \mathrm{u}_{\mathrm{xx}}=\frac{1}{\mathrm{H}} \frac{\partial}{\partial \mathrm{x}}\left(\mathrm{H}\left[\mathrm{D}_{\mathrm{xx}}+\frac{\Lambda_{\mathrm{x}}^{2}}{12}\left|\frac{\partial \mathrm{U}}{\partial \mathrm{x}}\right|\right]\right) \\
& \mathrm{u}_{\mathrm{xy}}=\frac{1}{\mathrm{H}} \frac{\partial}{\partial \mathrm{x}}\left(\mathrm{H}\left[\mathrm{D}_{\mathrm{xy}}+\frac{\Lambda_{\mathrm{y}}^{2}}{12}\left|\frac{\partial \mathrm{U}}{\partial \mathrm{y}}\right|\right]\right) \\
& \mathrm{u}_{\mathrm{xt}}=\frac{1}{\mathrm{H}} \frac{\partial}{\partial \mathrm{x}}\left(\mathrm{H} \frac{\Lambda_{\mathrm{t}}^{2}}{12}\left|\frac{\partial \mathrm{U}}{\partial \mathrm{t}}\right|\right)
\end{aligned}
$$

$$
\begin{gathered}
\mathrm{u}_{\mathrm{yx}}=\frac{1}{\mathrm{H}} \frac{\partial}{\partial \mathrm{y}}\left(\mathrm{H}\left[\mathrm{D}_{\mathrm{yx}}+\frac{\Lambda_{\mathrm{x}}^{2}}{12}\left|\frac{\partial \mathrm{V}}{\partial \mathrm{x}}\right|\right]\right) \\
\mathrm{u}_{\mathrm{yy}}=\frac{1}{\mathrm{H}} \frac{\partial}{\partial \mathrm{y}}\left(\mathrm{H}\left[\mathrm{D}_{\mathrm{yy}}+\frac{\Lambda_{\mathrm{y}}^{2}}{12}\left|\frac{\partial \mathrm{V}}{\partial \mathrm{y}}\right|\right]\right) \\
\mathrm{u}_{\mathrm{yt}}=\frac{1}{\mathrm{H}} \frac{\partial}{\partial \mathrm{y}}\left(\mathrm{H} \frac{\Lambda_{\mathrm{t}}^{2}}{12}\left|\frac{\partial \mathrm{V}}{\partial \mathrm{t}}\right|\right)
\end{gathered}
$$

$\mathrm{Na}$ Equação (4), as velocidades $\mathrm{u}_{\mathrm{xx}}, \mathrm{u}_{\mathrm{xy}}, \mathrm{u}_{\mathrm{xt}}$, $u_{y x}, u_{y y}$ e $u_{y t}$ são chamadas de "velocidades difusivas", representando a parcela referente às misturas difusivas.

Considerando que as reações cinéticas são de primeira ordem, podendo ser escritas como $\Sigma \mathrm{R}=-\mathrm{KC}$, sendo que $\mathrm{K}$ pode ser uma constante de decaimento ou de crescimento, dependendo do sinal considerado, tem-se então a equação de transporte de um escalar dada por:

$$
\begin{aligned}
& P_{t} \frac{\partial C}{\partial t}+P_{x} \frac{\partial C}{\partial x}+P_{y} \frac{\partial C}{\partial y}=P_{x x} \frac{\partial C^{2}}{\partial x^{2}}+P_{y y} \frac{\partial C^{2}}{\partial y^{2}}+ \\
& P_{x y} \frac{\partial}{\partial x}\left(\frac{\partial C}{\partial y}\right)+P_{y x} \frac{\partial}{\partial y}\left(\frac{\partial C}{\partial x}\right)+P_{x t} \frac{\partial}{\partial x}\left(\frac{\partial C}{\partial t}\right)+ \\
& P_{y t} \frac{\partial}{\partial y}\left(\frac{\partial C}{\partial t}\right)-K C
\end{aligned}
$$

onde:

$$
\begin{gathered}
P_{t}=1-u_{x t}-v_{y t}, P_{x}=U-u_{x x}-v_{y x} \\
P_{y}=V-u_{x y}-v_{y y}, P_{x x}=\frac{T_{x x}}{H}, P_{x y}=\frac{T_{x y}}{H}, \\
P_{x t}=\frac{T_{x t}}{H}, P_{y x}=\frac{T_{y x}}{H}, P_{y y}=\frac{T_{y y}}{H} \text { e } P_{y t}=\frac{T_{y t}}{H}
\end{gathered}
$$

\section{Condições de contorno}

Para que o problema definido pela Equação (5) seja bem posto, é necessário estabelecer condições iniciais e de contorno apropriadas. No modelo de transporte tem-se as condições de contorno de terra e aberto. Em todos estes contornos há condição de fluxo advectivo e difusivo segundo a direção normal. Esta condição pode ser genericamente escrita como: 


$$
\mathrm{U}_{\mathrm{N}} \mathrm{C}-\left(\mathrm{D}_{\mathrm{N}}+\frac{\Lambda_{\mathrm{N}}^{2}}{12}\left|\frac{\partial \mathrm{U}_{\mathrm{N}}}{\partial \mathrm{x}_{\mathrm{N}}}\right|\right) \frac{\partial \mathrm{C}}{\partial \mathrm{x}_{\mathrm{N}}}=\mathrm{f}_{\mathrm{N}}^{*}
$$

onde o subscrito $\mathrm{N}$ representa a direção normal e $\mathrm{f}_{\mathrm{N}}^{*}$ o fluxo normal prescrito na fronteira.

Em geral, ao longo dos contornos de terra, $\mathrm{U}_{\mathrm{N}}$ e $\mathrm{f}_{\mathrm{N}}^{*}$ são nulos em todos os nós. Neste caso, a equação resolvida nos nós de contorno de terra será:

$$
\frac{\partial \mathrm{C}}{\partial \mathrm{x}_{\mathrm{N}}}=0
$$

Ao longo da fronteira aberta é usual desconsiderar o fluxo difusivo. Em casos de efluxo, isto é, quando o transporte é para fora do domínio, o modelo resolve somente os termos advectivos, desprezando os termos difusivos, como se segue:

$$
\frac{\partial \mathrm{C}}{\partial \mathrm{t}}+\mathrm{U} \frac{\partial \mathrm{C}}{\partial \mathrm{x}}+\mathrm{V} \frac{\partial \mathrm{C}}{\partial \mathrm{y}}=\Sigma \mathrm{R}
$$

Ainda nas fronteiras abertas, e no caso de afluxo, quando a substância ingressa no domínio, a seguinte condição é considerada (Rosman, 2000):

Enquanto $\left(t-t_{0}\right) \leq \tau$ :

$$
\mathrm{C}=\mathrm{C}_{\mathrm{o}}+\frac{\mathrm{C}^{*}-\mathrm{C}_{\mathrm{o}}}{2}\left[1-\cos \left(\frac{\pi\left(\mathrm{t}-\mathrm{t}_{\mathrm{o}}\right)}{\tau}\right)\right]
$$

Quando $\left(t-t_{0}\right)>\tau$ :

$$
\mathrm{C}=\mathrm{C}^{*}
$$

onde $C^{*}$ representa o valor da concentração prescrita, $C_{o}$ é o valor da concentração calculada no ponto de contorno no instante $t_{0}$, ou seja, no momento de inversão da situação de efluxo para afluxo e $\tau$ é o período de transição, dado pelo modelador, baseado nos dados pretéritos. Usualmente este valor varia entre 0.5 e 1.0 hora. A visualização deste efeito pode ser observada na Figura 1.

\section{MODELO NUMÉRICO}

O esquema numérico utilizado para o modelo de transporte usa elementos finitos na discretização espacial e diferenças finitas na discretização temporal. Para tanto, é preciso escrever a equação na formulação residual ponderada e depois discretizar temporalmente.

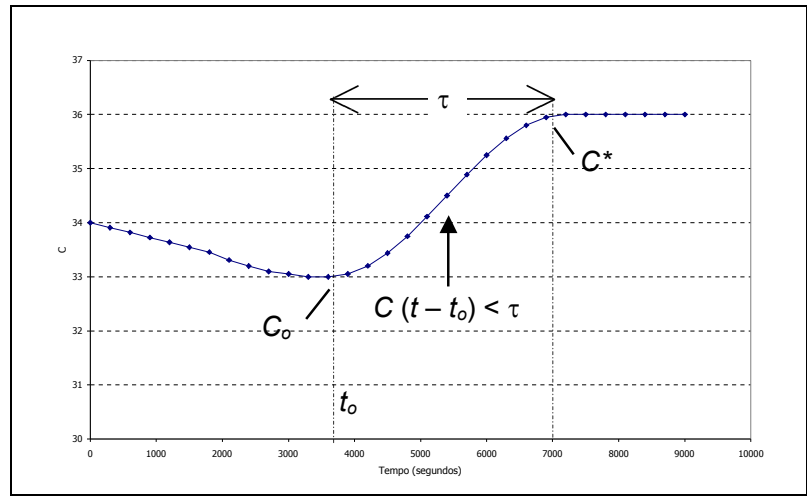

Figura 1. Visualização da condição de contorno de afluxo, usando a concentração de sal como exemplo.

A formulação residual ponderada da equação de transporte pode ser escrita como:

$$
\begin{aligned}
& \int_{\Omega}\left\{\left(P_{t} \frac{\partial C}{\partial t}+P_{x} \frac{\partial C}{\partial x}+P_{y} \frac{\partial C}{\partial y}+K C\right) \delta C\right. \\
& +\left(P_{x x} \frac{\partial C}{\partial x}+P_{x y} \frac{\partial C}{\partial y}+P_{x t} \frac{\partial C}{\partial t}\right) \frac{\partial \delta C}{\partial x} \\
& \left.+\left(P_{y x} \frac{\partial C}{\partial x}+P_{y y} \frac{\partial C}{\partial y}+P_{y t} \frac{\partial C}{\partial t}\right) \frac{\partial \delta C}{\partial y}\right\} d \Omega \\
& -\int_{\Gamma} f_{N}^{*} \delta C d \Gamma=0
\end{aligned}
$$

onde $\delta C$ é a função de peso ou ponderação, $\Omega$ representa o domínio do modelo, $\Gamma$ o contorno do domínio e $\mathrm{f}_{\mathrm{N}}^{*}$ o fluxo na direção normal. Deve-se ter em mente que a condição de contorno essencial é representada pela prescrição da concentração e que o fluxo representa a condição de contorno natural.

\section{Discretização temporal}

Tendo como objetivo a obtenção da versão discretizada no tempo da equação de transporte, a seguinte notação foi empregada:

$\begin{array}{lll}\text { Valores em } t: & C(x, y, t) & C \\ \text { Valores em } t+\Delta t: & C(x, y, t+\Delta t) & C^{n+1} \\ \text { Valores interpolados no } & C(x, y, t+\Delta t / 2) & C^{\otimes} \\ \text { tempo } t+\Delta t / 2: & & \end{array}$

Empregando o método do fatoramento implícito (Cunha, 2000), obtém-se a versão discretizada no tempo da equação de transporte como se segue: 


$$
\begin{aligned}
& \int\left\{\left(2 \frac{P_{t}^{\otimes}}{\Delta t} C^{n+1}+P_{x_{i}}^{\otimes} \frac{\partial C^{n+1}}{\partial x_{i}}+K C^{n+1}\right) \delta C\right. \\
& +\left(P_{x_{i}}^{\otimes} \frac{\partial C^{n+1}}{\partial x_{i}}+\frac{2 P_{x t}^{\otimes}}{\Delta t} C^{n+1}\right) \frac{\partial \delta C}{\partial x} \\
& \left.+\left(P_{y_{i}}^{\otimes} \frac{\partial C^{n+1}}{\partial x_{i}}+\frac{2 P_{y t}^{\otimes}}{\Delta t} C^{n+1}\right) \frac{\partial \delta C}{\partial y}\right\} d \Omega= \\
& \int\left\{\left(2 \frac{P_{t}^{\otimes}}{\Delta t} C^{n}-P_{x_{i}}^{\otimes} \frac{\partial C^{n+1}}{\partial x_{i}}+K C^{n}\right) \delta C\right. \\
& -\left(P_{\mathrm{xx}_{i}}^{\otimes} \frac{\partial C^{n+1}}{\partial x_{i}}+\frac{2 P_{x t}^{\otimes}}{\Delta t} C^{n}\right) \frac{\partial \delta C}{\partial x} \\
& \left.-\left(P_{y_{\mathrm{y} x}}^{\otimes} \frac{\partial C^{n+1}}{\partial x_{i}}-\frac{2 P_{y t}^{\otimes}}{\Delta t} C^{n}\right) \frac{\partial \delta C}{\partial y}\right\} d \Omega \\
& -\int_{\Gamma}^{*} f_{N}^{*} \delta C d \Gamma
\end{aligned}
$$

onde os parâmetros podem ser escritos como:

$$
\begin{aligned}
& \mathrm{P}_{\mathrm{x}}^{\otimes}=\mathrm{U}^{\otimes}-\frac{1}{\mathrm{H}^{\otimes}} \frac{\partial}{\partial \mathrm{x}_{\mathrm{i}}}\left[\mathrm{H}^{\otimes}\left(\mathrm{D}_{\mathrm{x}_{\mathrm{i}} \mathrm{x}}^{\otimes}+\frac{\Lambda_{\mathrm{x}}^{2}}{12}\left|\frac{\partial \mathrm{U}_{\mathrm{i}}^{\otimes}}{\partial \mathrm{x}}\right|\right)\right] \\
& \mathrm{P}_{\mathrm{y}}^{\otimes}=\mathrm{V}^{\otimes}-\frac{1}{\mathrm{H}^{\otimes}} \frac{\partial}{\partial \mathrm{x}_{\mathrm{i}}}\left[\mathrm{H}^{\otimes}\left(\mathrm{D}_{\mathrm{x}_{\mathrm{i}} \mathrm{y}}^{\otimes}+\frac{\Lambda_{\mathrm{y}}^{2}}{12}\left|\frac{\partial \mathrm{U}_{\mathrm{i}}^{\otimes}}{\partial \mathrm{y}}\right|\right)\right] \\
& \mathrm{P}_{\mathrm{t}}^{\otimes}=1-\frac{1}{\mathrm{H}^{\otimes}} \frac{\partial}{\partial \mathrm{x}_{\mathrm{i}}}\left[\mathrm{H}^{\otimes}\left(\frac{\Lambda_{\mathrm{t}}^{2}}{12}\left|\frac{\partial \mathrm{U}_{\mathrm{i}}^{\otimes}}{\partial \mathrm{t}}\right|\right)\right] \\
& \mathrm{P}_{\mathrm{xx}}^{\otimes}=\mathrm{D}_{\mathrm{xx}}^{\otimes}+\frac{\Lambda_{\mathrm{x}}^{2}}{12}\left|\frac{\partial \mathrm{U}^{\otimes}}{\partial \mathrm{x}}\right| \\
& \mathrm{P}_{\mathrm{yy}}^{\otimes}=\mathrm{D}_{\mathrm{yy}}^{\otimes}+\frac{\Lambda_{\mathrm{y}}^{2}}{12}\left|\frac{\partial \mathrm{V}^{\otimes}}{\partial \mathrm{y}}\right| \\
& \mathrm{P}_{\mathrm{xy}}^{\otimes}=\mathrm{D}_{\mathrm{xy}}^{\otimes}+\frac{\Lambda_{\mathrm{y}}^{2}}{12}\left|\frac{\partial \mathrm{U}^{\otimes}}{\partial \mathrm{y}}\right| \\
& \mathrm{P}_{\mathrm{yx}}^{\otimes}=\mathrm{D}_{\mathrm{yx}}^{\otimes}+\frac{\Lambda_{\mathrm{x}}^{2}}{12}\left|\frac{\partial \mathrm{V}^{\otimes}}{\partial \mathrm{x}}\right| \\
& \mathrm{P}_{\mathrm{xt}}^{\otimes}=\frac{\Lambda_{\mathrm{t}}^{2}}{12}\left|\frac{\partial \mathrm{U}^{\otimes}}{\partial \mathrm{t}}\right| \\
& \mathrm{P}_{\mathrm{yt}}^{\otimes}=\frac{\Lambda_{\mathrm{t}}^{2}}{12}\left|\frac{\partial \mathrm{V}^{\otimes}}{\partial \mathrm{t}}\right|
\end{aligned}
$$

\section{Discretização espacial}

$\mathrm{Na}$ discretização espacial são empregados elementos finitos quadráticos Lagrangeanos subparamétricos, sendo a geometria do elemento representada por polinômios Lagrangeanos lineares, enquanto a concentração e os parâmetros do domínio são definidos por polinômios Lagrangeanos quadráticos. Os elementos utilizados são os quadrangulares de 9 nós e os triangulares de 6 nós. Detalhes sobre a formulação de elementos finitos podem ser encontrados em (Zienkiewicz \& Morgan, 1982).

Aplicando a formulação de Galerkin para a equação de transporte e considerando $\varphi$ o conjunto de funções de interpolação dos elementos, os termos da equação podem ser escritos para cada nó $\mathrm{j}$ como:

$$
\begin{aligned}
& \sum_{\mathrm{e}=1}^{N E} \sum_{\mathrm{j}=1}^{N N}\left\{\mathrm{~W}_{\mathrm{ij}} \mathrm{C}_{\mathrm{j}}^{\mathrm{n}+1}\right\}= \\
& \sum_{\mathrm{e}=1}^{N E} \sum_{\mathrm{j}=1}^{N N}\left\{\mathrm{Z}_{\mathrm{ij}} \mathrm{C}_{\mathrm{ij}}\right\}+\left(\mathrm{f}_{\mathrm{N}}^{* \mathrm{n}=1}+\mathrm{f}_{\mathrm{N}}^{* \mathrm{n}}\right)_{\mathrm{j}} \int \varphi_{\Gamma} \varphi_{\mathrm{i}} \varphi_{\mathrm{j}} \mathrm{d} \Gamma
\end{aligned}
$$

onde:

$$
\begin{gathered}
W_{i j}=(A+B+C+D+E+F+G+H+I+J)_{i j} \\
Z_{i j}=(A-B-C-D-E+F-G-H+I-J)_{i j}
\end{gathered}
$$

sendo as matrizes definidas como:

$$
\begin{aligned}
& \mathrm{A}_{\mathrm{ij}}=\int_{\Omega_{\mathrm{e}}}\left\{\frac{2}{\Delta \mathrm{t}} \varphi_{\mathrm{i}} \varphi_{\mathrm{j}} \sum_{\mathrm{k}=1}^{\mathrm{NN}} \mathrm{P}_{\mathrm{t}_{\mathrm{k}}}^{\otimes} \varphi_{\mathrm{k}}\right\} \mathrm{d} \Omega_{\mathrm{e}} \\
& \mathrm{B}_{\mathrm{ij}}=\int_{\Omega_{\mathrm{e}}}\left\{\varphi_{\mathrm{i}} \frac{\partial \varphi_{\mathrm{j}}}{\partial \mathrm{x}} \sum_{\mathrm{k}=1}^{\mathrm{NN}} \mathrm{P}_{\mathrm{x}_{\mathrm{k}}}^{\otimes} \varphi_{\mathrm{k}}\right\} \mathrm{d} \Omega_{\mathrm{e}} \\
& \mathrm{C}_{\mathrm{ij}}=\int_{\Omega_{\mathrm{e}}}\left\{\varphi_{\mathrm{i}} \frac{\partial \varphi_{\mathrm{j}}}{\partial \mathrm{y}} \sum_{\mathrm{k}=1}^{\mathrm{N} N} \mathrm{P}_{\mathrm{y}_{\mathrm{k}}}^{\otimes} \varphi_{\mathrm{k}}\right\} \mathrm{d} \Omega_{\mathrm{e}} \\
& \mathrm{D}_{\mathrm{ij}}=\int_{\Omega_{\mathrm{e}}}\left\{\frac{\partial \varphi_{\mathrm{i}}}{\partial \mathrm{x}} \frac{\partial \varphi_{\mathrm{j}}}{\partial \mathrm{x}} \sum_{\mathrm{k}=1}^{\mathrm{NN}} \mathrm{P}_{\Omega_{\mathrm{e}}}^{\otimes}\left\{\frac{\partial \varphi_{\mathrm{i}}}{\partial \mathrm{x}} \frac{\partial \varphi_{\mathrm{j}}}{\partial \mathrm{y}} \sum_{\mathrm{k}=1}^{\mathrm{NN}} \mathrm{P}_{\mathrm{xy}} \varphi_{\mathrm{k}} \varphi_{\mathrm{k}}\right\} \mathrm{d} \Omega_{\mathrm{e}}\right. \\
& \mathrm{F}_{\mathrm{ij}}=\int_{\Omega_{\mathrm{e}}}\left\{\frac{2}{\Delta \mathrm{t}} \frac{\partial \varphi_{\mathrm{i}}}{\partial \mathrm{x}} \varphi_{\mathrm{j}} \sum_{\mathrm{k}=1}^{\mathrm{NN}} \mathrm{P}_{\mathrm{xt}} \otimes \varphi_{\mathrm{k}}\right\} \mathrm{d} \Omega_{\mathrm{e}}
\end{aligned}
$$




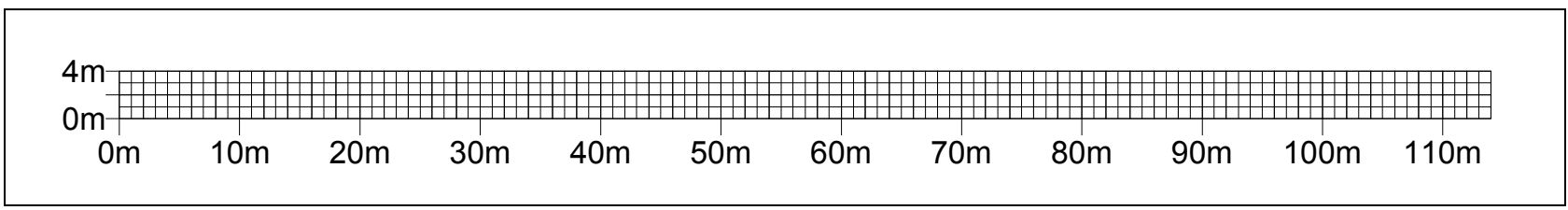

Figura 2. Malha usada na discretização do canal. Há 114 elementos e um total de 575 nós igualmente espaçados $(\Delta x=\Delta y=1,0 \mathrm{~m})$.

$$
\begin{gathered}
\mathrm{G}_{\mathrm{ij}}=\int_{\Omega_{\mathrm{e}}}\left\{\frac{\partial \varphi_{\mathrm{i}}}{\partial \mathrm{y}} \frac{\partial \varphi_{\mathrm{j}}}{\partial \mathrm{x}} \sum_{\mathrm{k}=1}^{\mathrm{NN}} \mathrm{P}_{\mathrm{yx}}^{\otimes} \varphi_{\mathrm{k}}\right\} \mathrm{d} \Omega_{\mathrm{e}} \\
\mathrm{H}_{\mathrm{ij}}=\int_{\Omega_{\mathrm{e}}}\left\{\frac{\partial \varphi_{\mathrm{i}}}{\partial \mathrm{y}} \frac{\partial \varphi_{\mathrm{j}}}{\partial \mathrm{y}} \sum_{\mathrm{k}=1}^{\mathrm{N} N} \mathrm{P}_{\mathrm{yy}}^{\otimes} \varphi_{\mathrm{k}}\right\} \mathrm{d} \Omega_{\mathrm{e}} \\
\mathrm{I}_{\mathrm{ij}}=\int_{\Omega_{\mathrm{e}}}\left\{\frac{2}{\Delta \mathrm{t}} \frac{\partial \varphi_{\mathrm{i}}}{\partial \mathrm{y}} \varphi_{\mathrm{j}} \sum_{\mathrm{k}=1}^{\mathrm{NN}} \mathrm{P}_{\mathrm{yt}_{\mathrm{k}}}^{\otimes} \varphi_{\mathrm{k}}\right\} \mathrm{d} \Omega_{\mathrm{e}} \\
\mathrm{J}_{\mathrm{ij}}=\int_{\Omega_{\mathrm{e}}}\left\{\mathrm{K} \varphi_{\mathrm{i}} \varphi_{\mathrm{j}}\right\} \mathrm{d} \Omega_{\mathrm{e}}
\end{gathered}
$$

Nas expressões acima, NE representa o número de elementos em que o domínio foi subdivido e NN o número de pontos nodais de cada elemento, no caso 9 ou 6, já que foram implementados dois elementos no FIST-ADE: os quadrangulares e os triangulares.

O sistema mostrado na Equação (13) é resolvido através do método iterativo de solução GMRES (Generalized Minimum Residual Method), usando precondicionadores do tipo ILUT (Incomplete LU factorization with dual truncation strategy), desenvolvido por Saad (1995). Maiores detalhes sobre a implementação do GMRES na solução de sistemas lineares podem ser encontrados em Jorge (1999).

\section{VALIDAÇÃO DO MODELO}

Os modelos numéricos precisam ser validados e testados quanto à sua estabilidade, consistência e precisão. Usualmente estes testes são feitos comparando as respostas obtidas pelos modelos com soluções analíticas conhecidas ou valores medidos em laboratório. Para validar o código computacional do FIST-ADE foram realizados dois testes, resolvendo de forma numérica problemas com soluções analíticas conhecidas.

\section{Teste para uma fonte plana instantânea em canal retilíneo uniforme com velocidade zero}

Este teste corresponde ao lançamento instantâneo de uma quantidade de massa $M$ unifor-
Tabela 1. Parâmetros usados no modelo numérico.

\begin{tabular}{lc}
\hline Parâmetros & Valor \\
\hline $\mathrm{D}_{\mathrm{xx}}\left(\mathrm{m}^{2} / \mathrm{s}\right)$ & 4,0 \\
$\Delta \mathrm{t}(\mathrm{s})$ & 1,0 \\
Número de Peclèt & 0,0 \\
Número de Courant & 0,0 \\
$\gamma\left(\mathrm{D} \Delta \mathrm{t} / \Delta \mathrm{x}^{2}\right.$ - razão entre a difusão física & 4,0 \\
e a numérica) & \\
\hline
\end{tabular}

memente distribuída na seção transversal de um canal infinitamente longo, alinhado com a direção x. O canal tem uma seção transversal uniforme A. A substância em questão é conservativa, e portanto, não há reações cinéticas. A água no canal está em repouso, logo $U_{i}$ é zero. Nestas condições, a equação de transporte advectivo-difusivo bidimensional, Equação (1), reduz-se a:

$$
\frac{\partial C}{\partial t}=D_{x x} \frac{\partial^{2} C}{\partial x^{2}}
$$

A Figura 2 mostra a geometria do canal considerado, bem como a malha de discretização aplicada. A profundidade do canal é uniforme e igual a $5,0 \mathrm{~m}$, resultando uma seção transversal A $=20,0 \mathrm{~m}$.

As condições de contorno para este problema constituem na imposição de:

- $C(x, t)=0$ para valores de $x$ tendendo a $\pm \infty$.

Condições iniciais:

- $C(x=57,0 m, t=0)=M /(A \Delta x) e$

- $C(x \neq 57,0 m, t=0)=0,0$.

A solução geral para este problema pode ser encontrada em Fischer (1979), e lê-se:

$$
C(x, t)=\frac{M}{A \sqrt{4 \pi D_{x x} t}} \exp \left(\frac{-\left(x-x_{0}\right)^{2}}{4 D_{x x} t}\right)
$$

A simulação numérica para este teste utilizou os parâmetros listados na Tabela 1. O lança- 


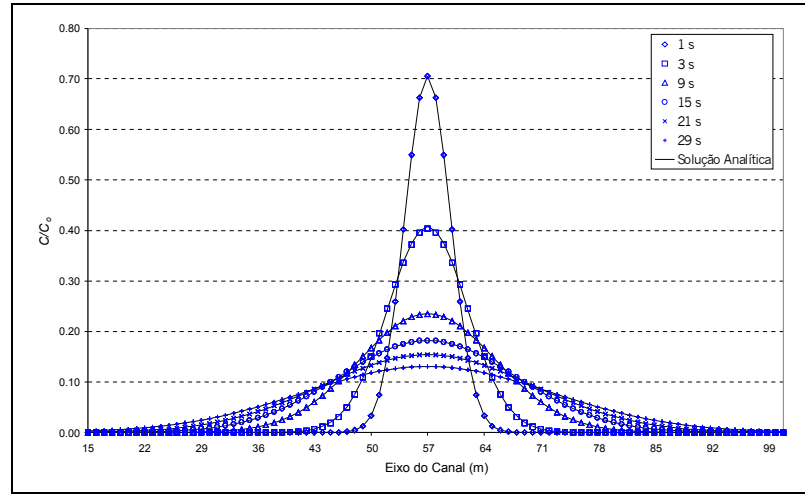

Figura 3. Comparação entre os resultados do FIST-ADE com a solução analítica em diferentes instantes, considerando IDC $=1$.

mento instantâneo foi feito em $x=57,0 \mathrm{~m}$. Evidentemente o canal numérico não é infinito e, conseqüentemente, não é possível prescrever as condições de contorno teóricas. Foi especificada, como condição de contorno, vazão normal igual a zero em todos os pontos do contorno. Sendo assim, a simulação numérica concorda com a solução analítica até o instante em que a reflexão nas extremidades do canal se torna significativa.

Para verificar os resultados do modelo, os valores calculados pelo modelo numérico, com e sem interface dissipativa, foram comparados com a solução analítica para a concentração. A interface dissipativa tem importante papel em algumas simulações porque os esquemas numéricos usados no modelo de transporte Euleriano são esquemas não dissipativos. Tais esquemas numéricos tendem a gerar oscilações numéricas espúrias, as quais precisam ser controladas e removidas sem afetar os fenômenos de interesse de larga escala. Para maiores detalhes sobre a interface, procurar Rosman (2000) e Rosman \& Gobbi (1990).

O parâmetro de controle dessa interface dissipativa é IDC $=\mathrm{N}$, onde $\mathrm{N}$ é um número inteiro. Se $\mathrm{N}>0$, a interface é aplicada $\mathrm{N}$ vezes em cada intervalo de tempo. Se $N=0$, a interface não é aplicada. Se $\mathrm{N}<0$, a interface é aplicada uma vez a cada $\mathrm{N}$ intervalos de tempo.

Os resultados do teste são apresentados na Figura 3. Como pode ser observado da comparação entre os resultados numéricos, obtidos pelo FIST-ADE, e a solução analítica, uma boa concordância foi obtida com o uso da interface dissipativa (IDC $=1)$.

\section{Testes para uma fonte plana em um canal retilíneo uniforme com velocidade uniforme}

Estes testes correspondem a uma fonte plana contínua com uma quantidade de massa igual a

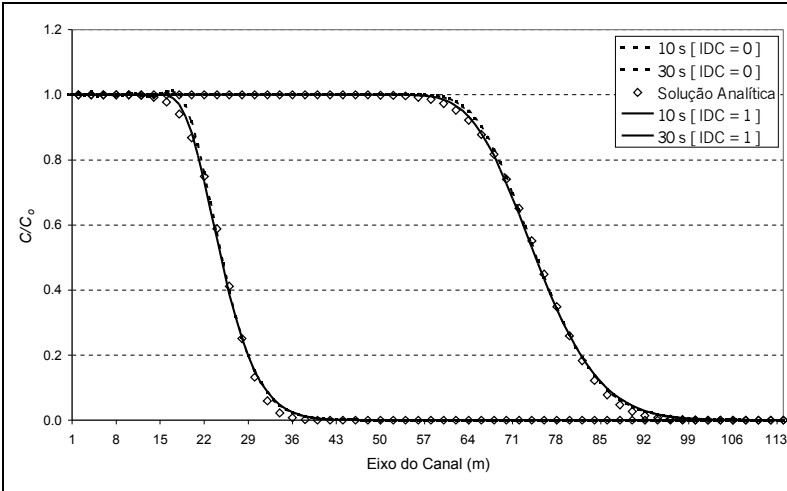

Caso \#1.

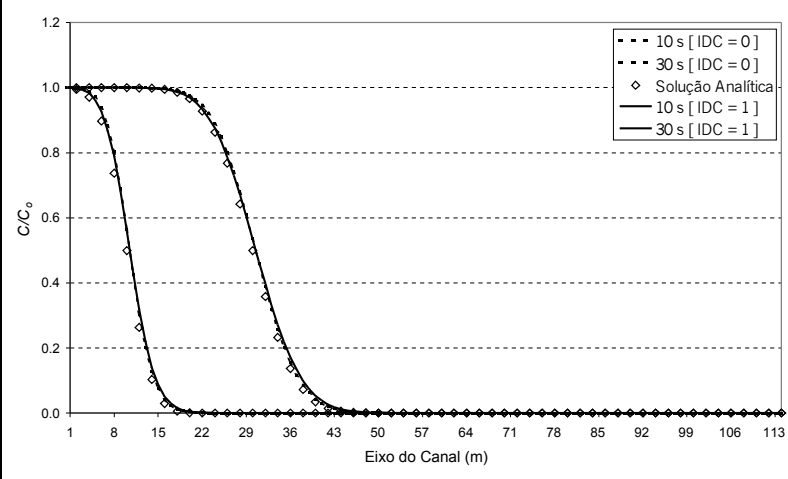

Caso \#2.

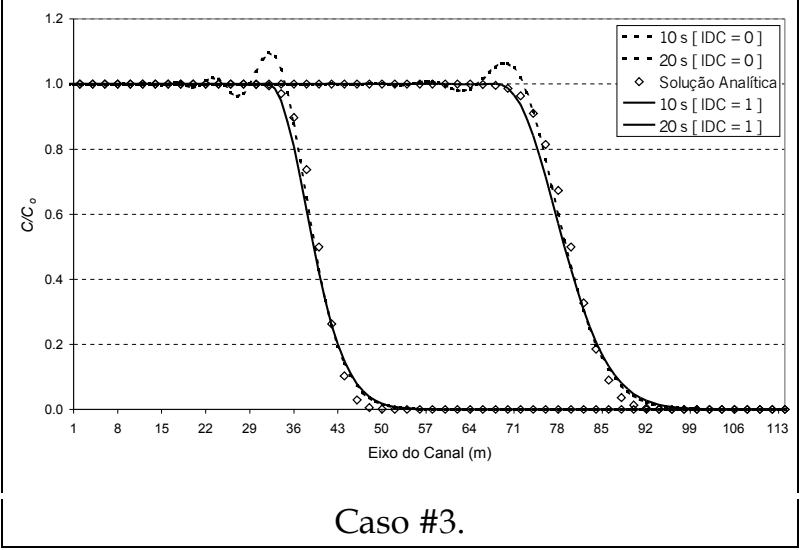

Figura 4. Comparação entre os resultados do FIST-ADE e as soluções analíticas para os diferentes casos.

M, uniformemente distribuída na seção transversal uniforme $\mathrm{A}$ posicionada em $\mathrm{x}=0,0$. A água escoa no canal com uma velocidade constante U. Não foi considerado, para efeito de simulação, qualquer reação cinética, ou seja, a substância é conservativa. Segundo estas condições, a equação de transporte pode ser reduzida a:

$$
\frac{\partial \mathrm{C}}{\partial \mathrm{t}}+\mathrm{U} \frac{\partial \mathrm{C}}{\partial \mathrm{x}}=\mathrm{D}_{\mathrm{xx}} \frac{\partial^{2} \mathrm{C}}{\partial \mathrm{x}^{2}}
$$


As condições de contorno do problema são:

- $C(0, t)=M /(A \Delta x)$;

- fluxo normal nulo ao longo das paredes.

As condições iniciais correspondem a:

- $\mathrm{C}(0,0)=\mathrm{M} /(\mathrm{A} \Delta \mathrm{x}) \mathrm{e}$;

- $C(x \neq 0,0)=0,0$.

A solução analítica para o problema proposto pode ser encontrada em Fischer (1979) e é dada por:

$$
\frac{C}{C_{0}}=\frac{1}{2}\left[1-\operatorname{erf} \frac{(x-U t)}{\sqrt{4 D_{x x} t}}\right]
$$

onde $\mathrm{C}_{\mathrm{o}}$ é uma concentração de referência.

O canal usado para o lançamento é o mesmo do teste anterior, mostrado na Figura 2. Foram desenvolvidos três testes numéricos, usando diversos parâmetros de simulação, observando assim os efeitos, sobre a solução numérica, de diferentes coeficientes de difusão para distintas condições hidrodinâmicas do corpo receptor. O objetivo é verificar o comportamento do modelo diante de condições adversas como, por exemplo, altos números de Pèclet e Courant. Os parâmetros usados estão mostrados na Tabela 2.

O esquema numérico usado no modelo apresenta efeitos de difusão numérica, ou seja, oscilações, que são tanto mais importantes quanto menor é a difusão física do problema. Sendo assim, a interface dissipativa foi utilizada para retirar as oscilações numéricas indesejáveis, sem o comprometimento da solução.

Na Figura 4 são mostrados os resultados numéricos obtidos pelo FIST-ADE e as soluções analíticas, para os diferentes casos, em dois instantes de tempo distintos. Pode-se observar que nos casos \#1 e \#2 os resultados numéricos apresentam boa concordância com a solução analítica, considerando ou não a interface dissipativa. No entanto, no caso \#1 pode-se afirmar que a presença desta interface melhora o resultado numérico, sem provocar abatimento da resposta. No caso \#3, a interface trabalha para retirar as oscilações numéricas apresentadas pela solução numérica, melhorando de forma significativa o resultado, sem abater a solução.

Como no teste anterior, foram feitas simulações usando ou não a interface dissipativa, com o
Tabela 2. Parâmetros utilizados na simulação numérica para uma fonte plana contínua com velocidade uniforme.

\begin{tabular}{lccc}
\hline Parâmetros & \multicolumn{3}{c}{ Casos } \\
& $\# 1$ & $\# 2$ & $\# 3$ \\
\hline $\mathrm{D}_{\mathrm{xx}}\left(\mathrm{m}^{2} / \mathrm{s}\right)$ & 2,0 & 0,5 & 1,0 \\
$\mathrm{U}(\mathrm{m} / \mathrm{s})$ & 2,0 & 4,0 & 2,5 \\
$\Delta \mathrm{t}(\mathrm{s})$ & 2,0 & 0,5 & 1,0 \\
Número de Peclèt & 1,0 & 8,0 & 2,5 \\
Número de Courant & 4,0 & 2,0 & 2,5 \\
$\gamma\left(\mathrm{D} \Delta \mathrm{t} / \Delta \mathrm{x}^{2}\right.$ - razão entre a & 4,0 & 0,25 & 1,0 \\
difusão física e a numérica) & & & \\
\hline
\end{tabular}

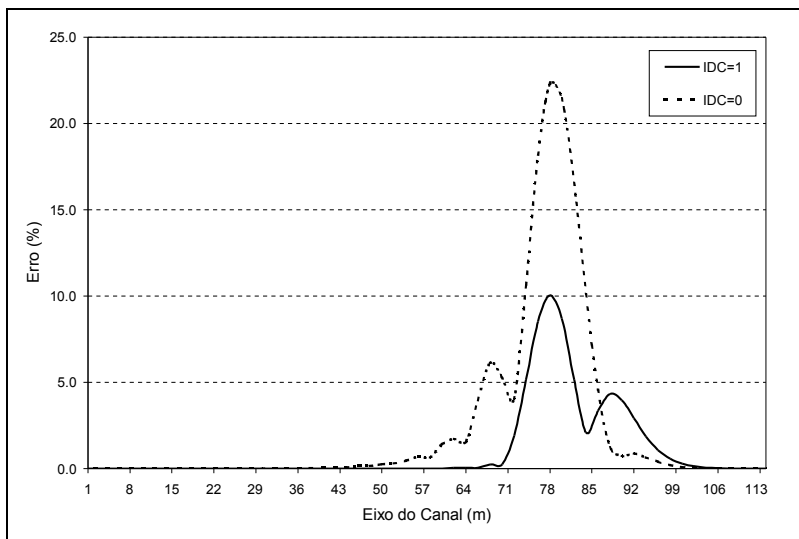

Figura 5. Erro médio quadrático para caso \#3 no instante $t=20 \mathrm{~s}$.

objetivo de controlar e remover as oscilações numéricas, sem afetar a resposta do problema (Vinzon, 1991). Se as oscilações não forem removidas, a solução numérica não é boa, como fica claramente demonstrado no caso \#3. Sendo assim, uma estimativa do erro médio quadrático foi realizada considerando os três casos em estudo. O erro médio quadrático pode ser definido como:

$$
\text { Erro }=\frac{1}{M} \int_{\Omega}\left[\left(C_{M}-C_{a}\right)^{2} d x d y\right]^{1 / 2}
$$

onde $\mathrm{C}_{\mathrm{M}}$ é a concentração calculada numericamente, $C_{a}$ é a concentração calculada analiticamente e $M$ é a massa total de escalar despejado.

A Figura 5 mostra o erro médio quadrático para caso \#3, com ou sem a interface dissipativa. Pode-se observar que o uso da interface diminui o erro médio quadrático ao longo de todo intervalo de simulação, melhorando bastante o resultado numérico (Oliveira et al., 2000). Nos demais casos, os erros médios quadráticos encontrados foram da 
ordem de $4 \%$, bem inferior ao do caso \#3. Nestes casos, a interface dissipativa altera muito pouco o padrão de distribuição dos erros ao longo dos intervalos de simulação, mostrando que a interface criada não compromete a solução numérica, e sim melhora o seu comportamento, removendo as oscilações numéricas espúrias.

\section{SIMULAÇÃO DO TRANSPORTE DE SAL NA BAÍA DE SEPETIBA}

A Baía de Sepetiba está localizada no Estado do Rio de Janeiro, próxima à região metropolitana da cidade do Rio de Janeiro. As conseqüências da expansão desta região trouxeram graves proble-

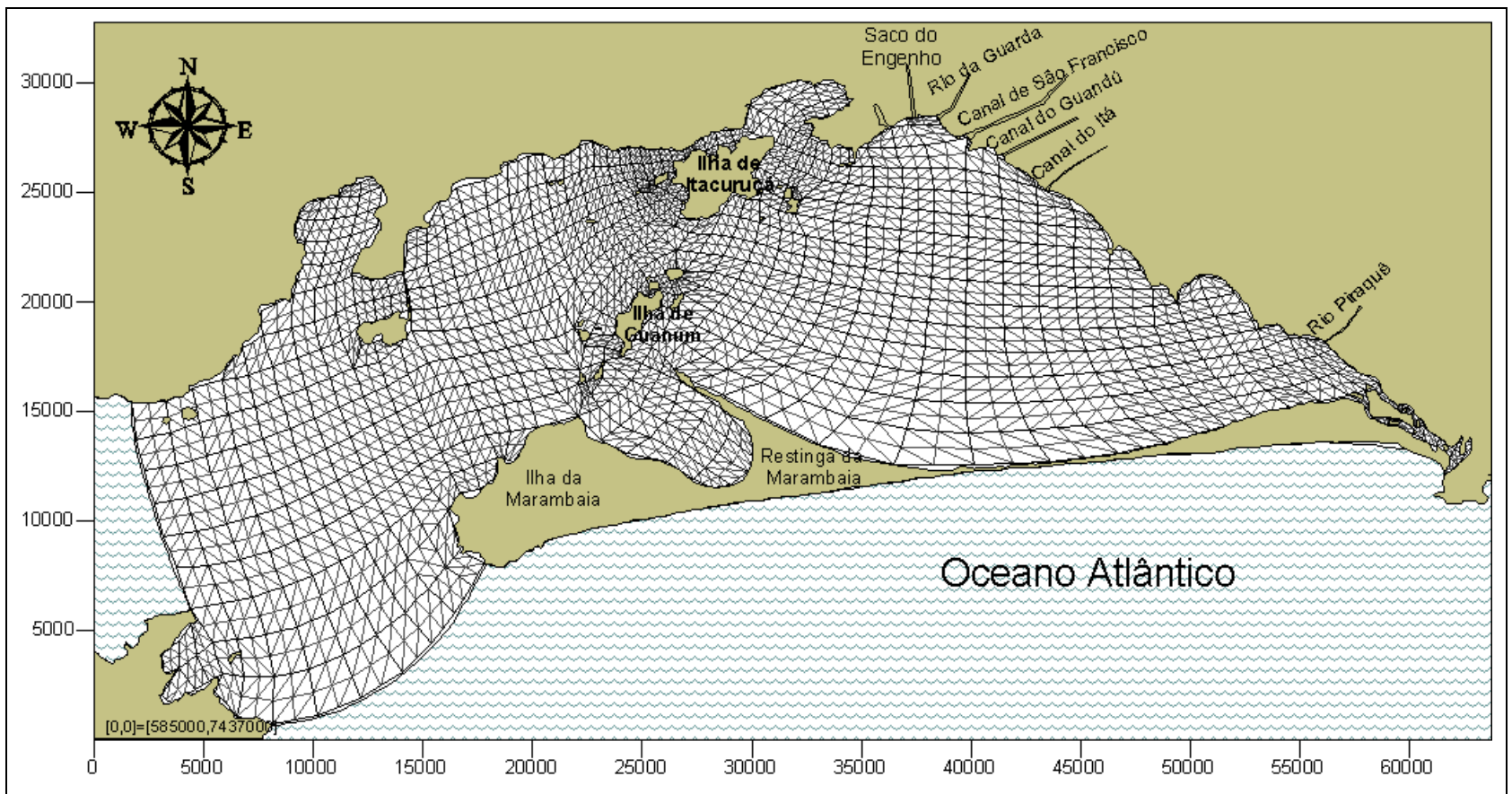

Figura 6. Domínio de modelagem para o sistema da Baía de Sepetiba, mostrando a malha em elementos finitos com 553 elementos e 2477 nós. As linhas mais grossas marcam os elementos. Os triângulos lineares interiores, conectando os nós de cada elemento, apenas auxiliam na visualização da densidade da malha.

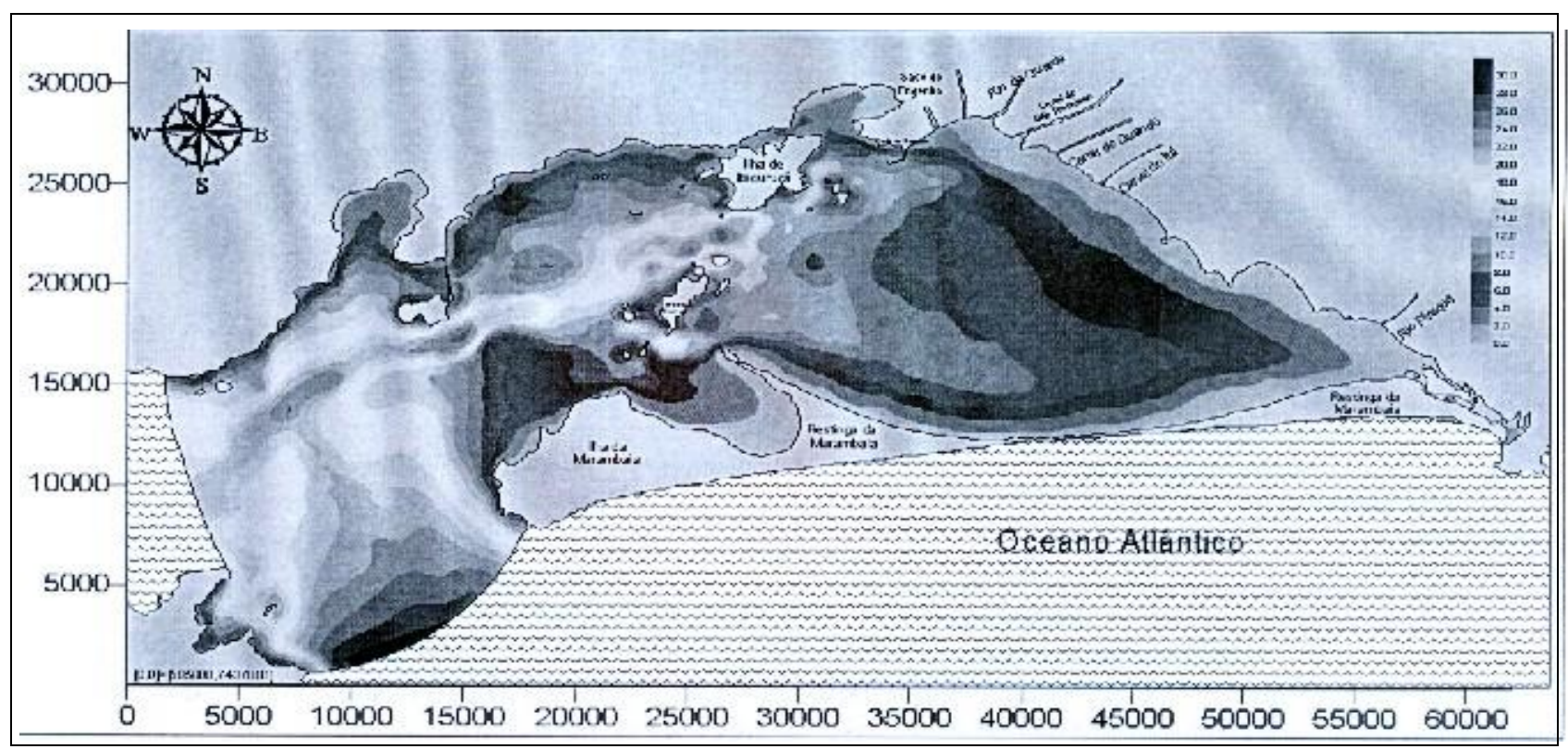

Figura 7. Visualização da topografia de fundo do domínio de modelagem conforme visto pela malha de discretização (Figura 6). 
mas ambientais a esta Baía e, no que se refere à qualidade da água, foram provocados principalmente pelo lançamento de esgotos sanitários e de resíduos sólidos urbanos. O Departamento de Saneamento e Saúde Ambiental da Escola Nacional de Saúde Pública/FIOCRUZ, juntamente com a Área de Engenharia Costeira e Oceanográfica
(AECO) da COPPE/UFRJ, desenvolvem um projeto conjunto com o objetivo de estudar a dispersão de alguns parâmetros de qualidade da água na Baía de Sepetiba, através do desenvolvimento de um modelo de qualidade da água considerando várias espécies. Neste contexto, a simulação de sal na baía é o primeiro passo no estudo de escalares passivos

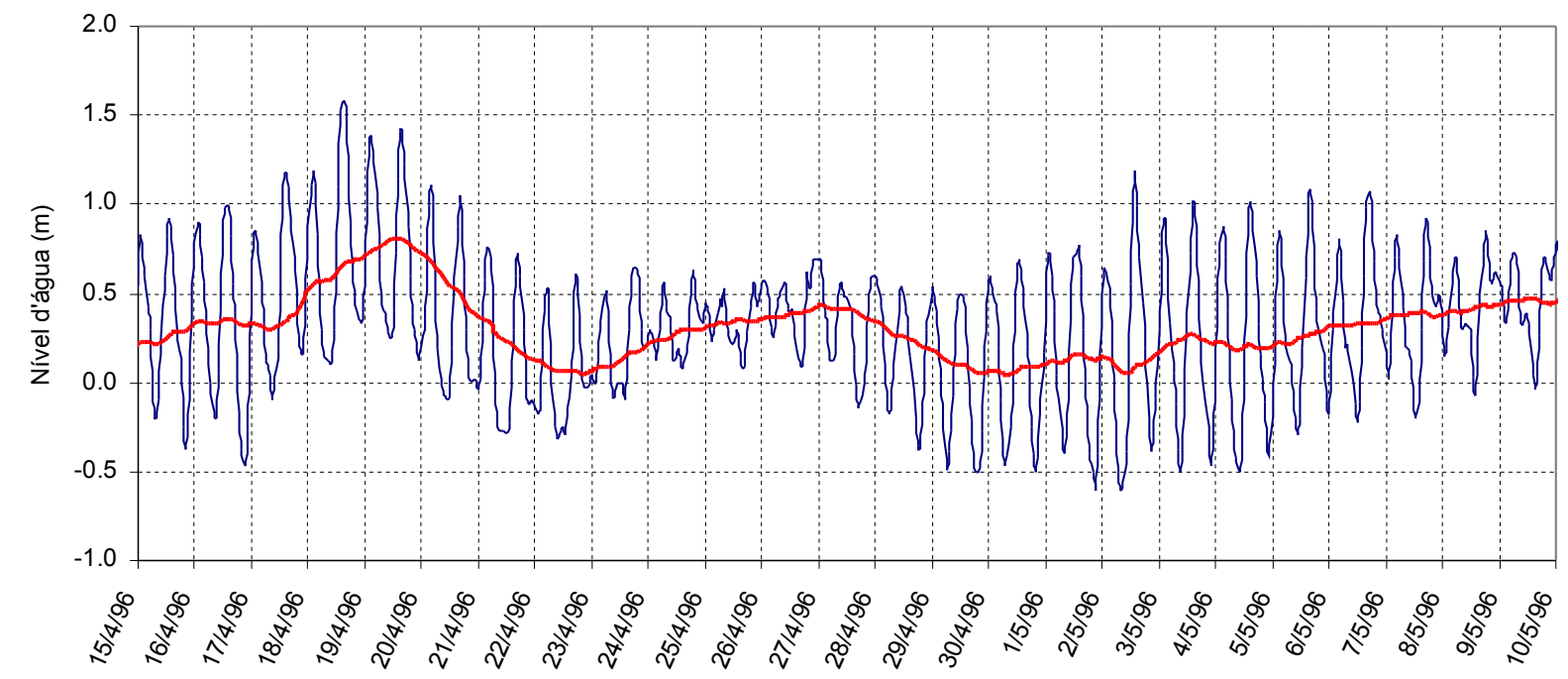

Figura 8. Curva de maré utilizada na modelagem, obtida a partir de dados do DHN na Ilha Guaíba. O nível zero corresponde ao nível médio do mar na região. A diferença entre o zero e o nível médio diário é a maré meteorológica.

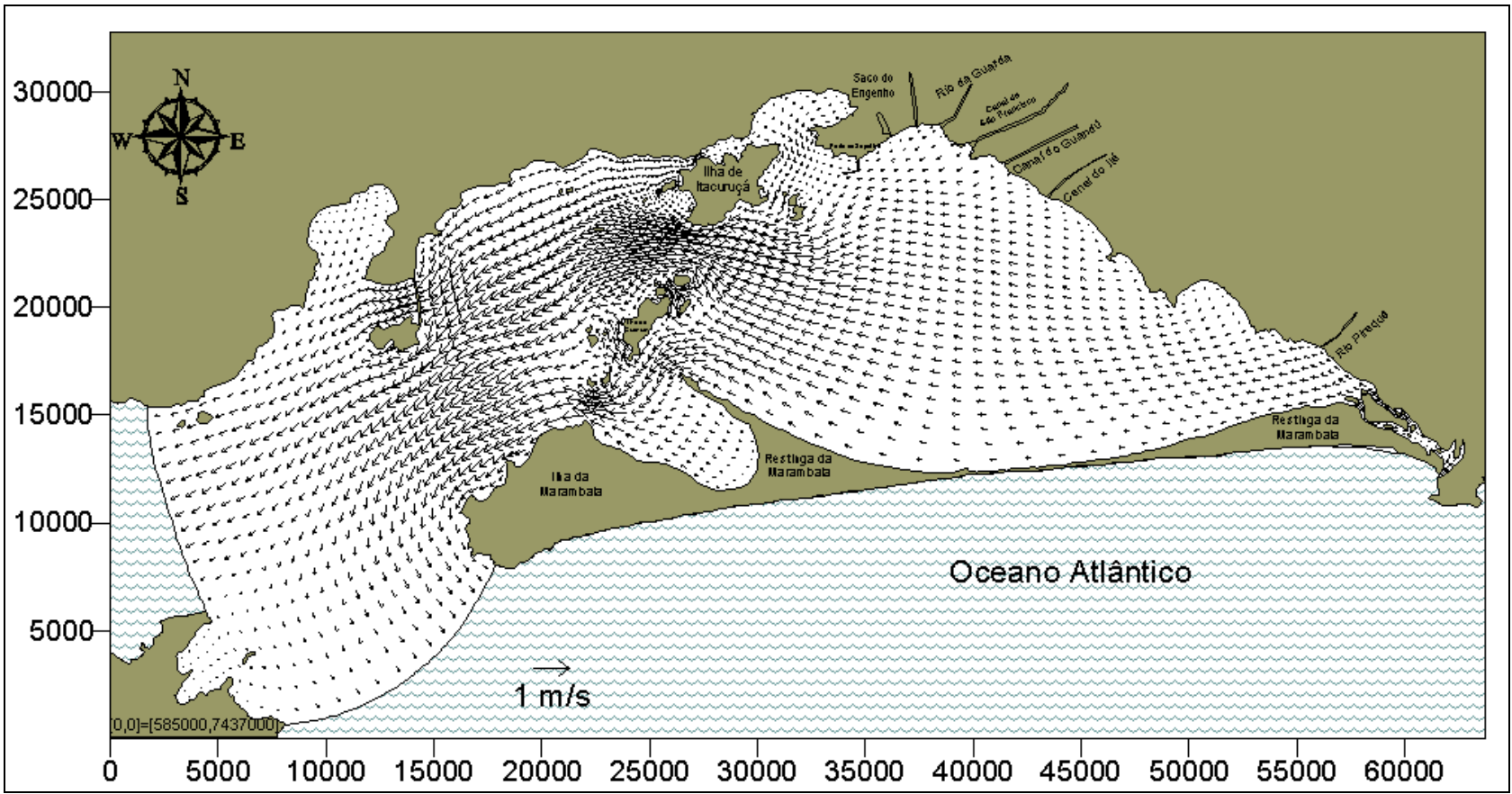

Figura 9. Campo de correntes de maré simuladas, em situação de sizígia, próximo ao instante de meia maré vazante (06:00 h de 03/05/96). Nas regiões em torno das ilhas há uma intensificação da velocidade de corrente e, na região mais interna da Baía, uma gradual diminuição das correntes. 
misturados na coluna d'água e distribuídos ao longo da Baía de Sepetiba.

Sendo assim, o objetivo desta simulação é mostrar a aplicação do FIST-ADE na modelagem de transporte de sal em corpos d'água reais e, futuramente, usá-lo como base no desenvolvimento de um modelo de qualidade de água considerando várias espécies. Os resultados mostrados aqui referem-se ao estágio inicial do trabalho, ilustrando as potencialidades do FIST-ADE para este tipo de aplicação.

A Baía de Sepetiba possui uma área de aproximadamente $305 \mathrm{~km}^{2}$. É um corpo de águas salinas e salobras, comunicando-se com o oceano Atlântico através de duas passagens: uma na parte oeste e outra menor, na parte leste, pelo canal que deságua na Barra de Guaratiba. Considerando que o interesse do estudo é a simulação do transporte de escalar dentro da Baía de Sepetiba, o domínio definido para o estudo compreende toda a Baía, com uma pequena extensão na região da Baía da Ilha Grande. Desta forma é possível estudar a circulação e o transporte ao longo da Baía considerando as influências das regiões vizinhas sobre a sua circulação. O domínio definido na modelagem é mostrado na Figura 6, onde também pode ser observada a malha de elementos finitos quadráticos usada na discretização do domínio, a partir de elementos quadrangulares sub-paramétricos Lagrangeanos. A batimetria da Baía, apresentada na Figura 7, foi obtida utilizando as cartas náuticas da DHN (Diretoria de Hidrografia e Navegação) n¹607, escala 1:80.000 e n 1622 , escala 1:40.122.

Na simulação do padrão de circulação hidrodinâmica foram consideradas as marés medidas na Ilha Guaíba entre os dias 15/04/96 e 10/05/96, fornecidas pela DHN, como o principal forçante, uma vez que a circulação é regida principalmente pelo afluxo e efluxo da maré (Nguyen et al., 1997). Neste período, através de um projeto de cooperação técnica entre a FEEMA/GTZ (Fundação Estadual de Engenharia do Meio Ambiente e Projeto de Cooperação Técnica Brasil-Alemanha), foram feitas medições de corrente em quatro pontos da Baía de Sepetiba. Estes dados foram utilizados para uma primeira calibração do padrão de circulação hidrodinâmica da Baía (Hsu et al., 1999). Uma descrição detalhada da calibração dos resultados hidrodinâmicos foge do escopo deste trabalho e não será apresentada.

Na Figura 8 é mostrada a curva de nível d'água medida na Ilha Guaíba. Pode-se observar uma condição de maré meteorológica típica de en-
Tabela 3. Vazões dos rios afluentes ao domínio da Baía de Sepetiba.

\begin{tabular}{lr}
\hline Rio & \multicolumn{1}{c}{ Vazão } \\
\hline Rio da Guarda & $19,1 \mathrm{~m}^{3} / \mathrm{s}$ \\
Canal de São Francisco & $187,0 \mathrm{~m}^{3} / \mathrm{s}$ \\
Canal do Guandú & $8,8 \mathrm{~m}^{3} / \mathrm{s}$ \\
Canal do Itá & $3,2 \mathrm{~m}^{3} / \mathrm{s}$ \\
Saco do Engenho & $0,5 \mathrm{~m}^{3} / \mathrm{s}$ \\
Rio Piraquê & $4,9 \mathrm{~m}^{3} / \mathrm{s}$ \\
\hline
\end{tabular}

trada de frente fria durante este período. Nota-se que no dia 19/04/96 o nível médio estava cerca de $75 \mathrm{~cm}$ acima do usual e vai aproximar-se do usual no dia 23/04/96.

Os dados de vento utilizados foram os medidos na região de Santa Cruz, próximo à Baía de Sepetiba. Considerou-se o campo de vento uniforme no espaço, mas variando ao longo do tempo, com valores definidos a cada hora. Foram considerados ainda seis rios afluentes na região modelada da Baía de Sepetiba. As vazões médias adotadas podem ser observadas na Tabela 3. A localização dos rios pode ser observada na Figura 6.

O padrão de circulação hidrodinâmica bidimensional, ou seja, considerando o campo de correntes promediadas na vertical, definido na Baía de Sepetiba, pode ser observado nas Figuras 9 a 11, dentro de um ciclo de maré.

O objetivo deste exemplo consiste apenas em uma comparação qualitativa entre os dados medidos em campo com o resultado obtido pelo modelo, já que os dados de maré e vento usados nesta modelagem estão referidos nas mesmas datas da campanha feita pela FEEMA/GTZ (Fundação Estadual de Engenharia do Meio Ambiente e Projeto de Cooperação Técnica Brasil-Alemanha), permitindo assim uma comparação qualitativa dos resultados.

Observando os resultados pode-se verificar que na parte mais interna da Baía, onde as profundidades são menores, as correntes são mais fracas, quando comparadas com as correntes geradas nos estreitamentos próximos às Ilhas. Nestas regiões, as correntes são mais intensas, favorecidas pela redução da área e pela presença de um canal natural de maior profundidade.

Como o FIST-ADE considera escalares passivos, a modelagem do padrão hidrodinâmico do corpo d'água e a modelagem do transporte do escalar são problemas desacoplados. Por esta razão, a correta simulação do padrão hidrodinâmico é deci- 
siva na simulação do transporte de sal ou de qualquer outro escalar. O sal normalmente é usado como um traçador, ou seja, como um escalar utilizado na calibração do modelo. Se a distribuição de sal for bem simulada, os demais escalares passivos que se encontram dissolvidos ao longo da coluna d'água e distribuídos por toda área da Baía também poderão ser corretamente simulados.

Na simulação do transporte de sal, os campos de velocidades e de elevação são dados pelo mo-

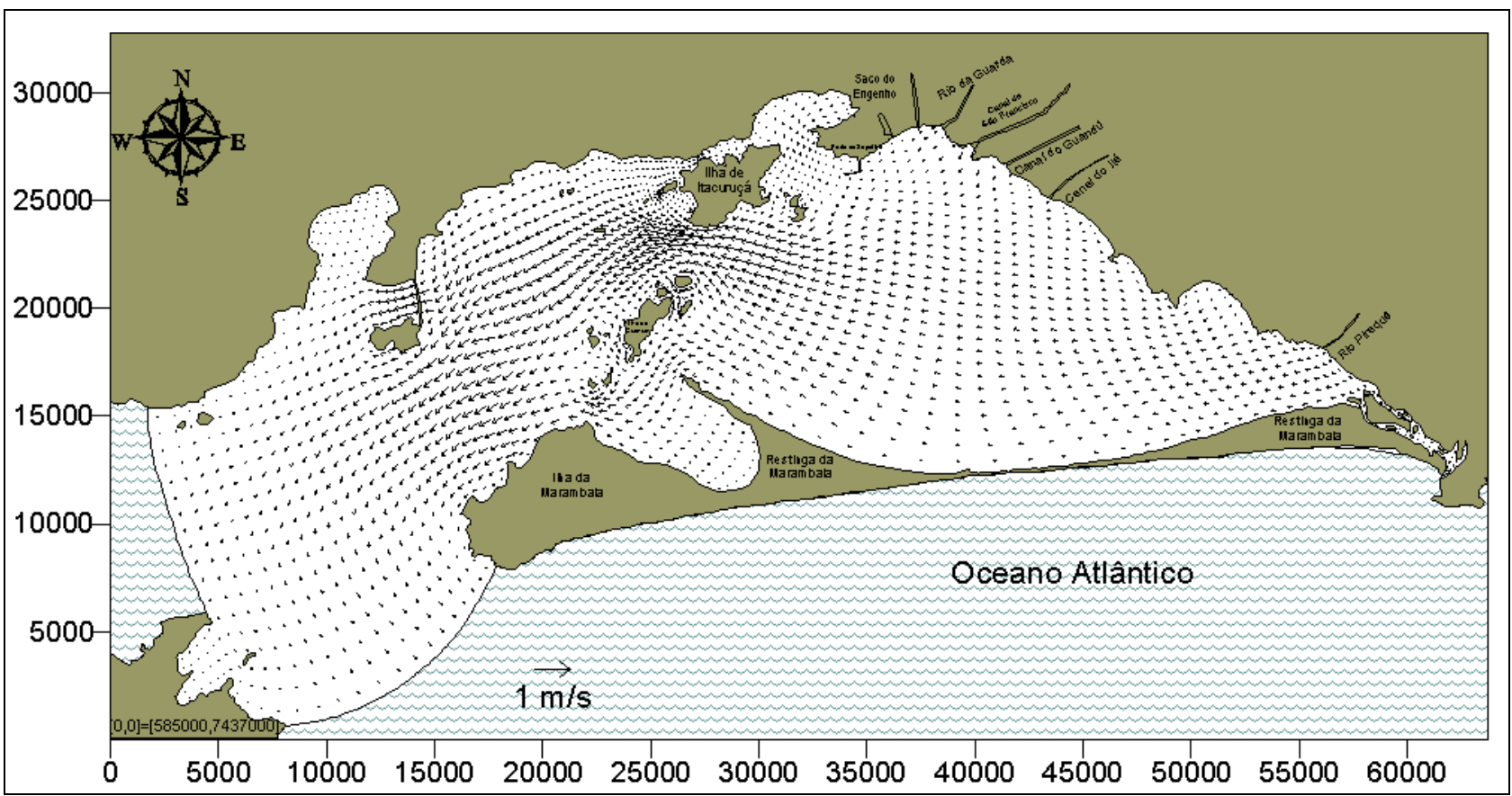

Figura 10. Campo de correntes de maré simuladas, em situação de sizígia, próximo ao instante de baixa-mar (09:00 h de 03/05/96). Note-se uma diminuição na velocidade das correntes, já que a escala utilizada é a mesma da Figura 9.

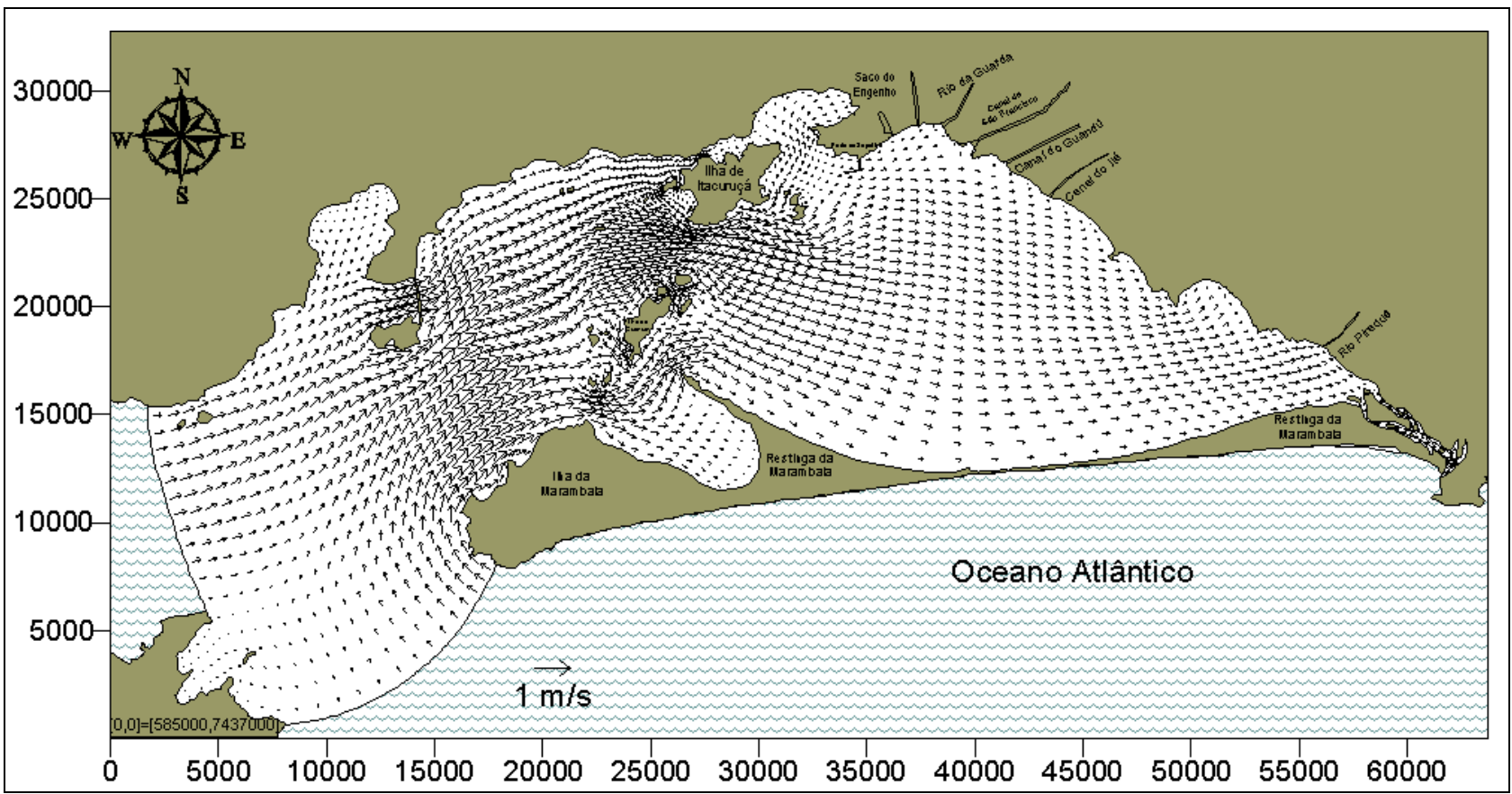

Figura 11. Campo de correntes de maré simuladas, em situação de sizígia, próximo ao instante de meia maré enchente (12:00 h de 03/05/96). Note-se que o padrão de circulação é bastante semelhante ao apresentado na Figura 9, com as direções das correntes invertidas. 
delo hidrodinâmico. As condições de contorno nos rios são descritas a partir do fluxo de água doce desses rios, considerando que o prisma de maré penetra rio acima e se mistura com o volume de água doce correspondente às vazões dos rios, den- tro do intervalo de tempo considerado na simulação. As vazões dos rios foram consideradas permanentes, já que as flutuações no intervalo simulado são muito pequenas. Sendo assim, os fluxos também foram tomados como permanentes e foram con-

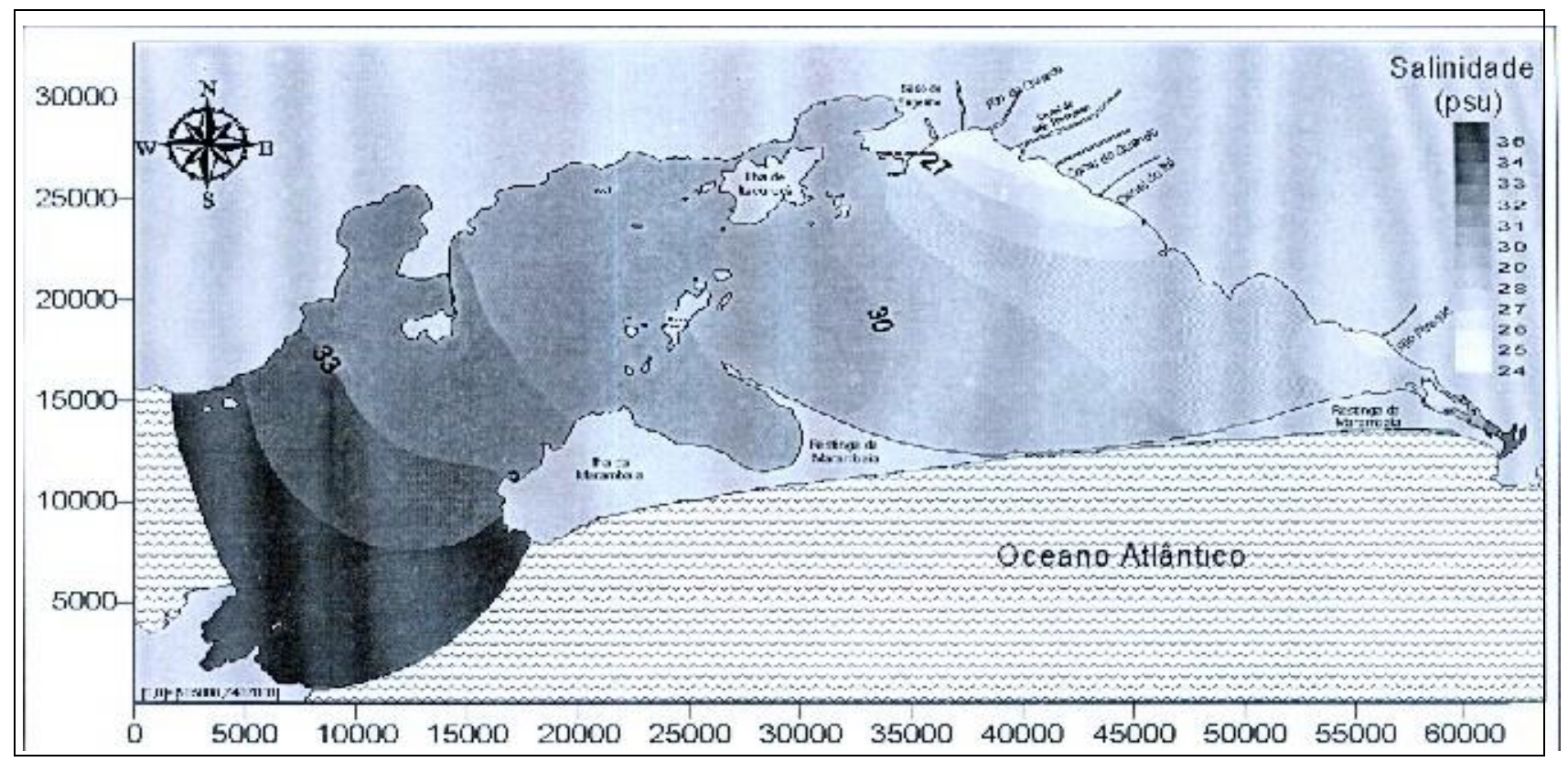

Figura 12. Distribuição de salinidade simulada com o FIST-ADE próxima ao instante de baixa-mar (09:00 h de 18/04/96), em situação de sizígia, considerando maré astronômica e maré meteorológica.

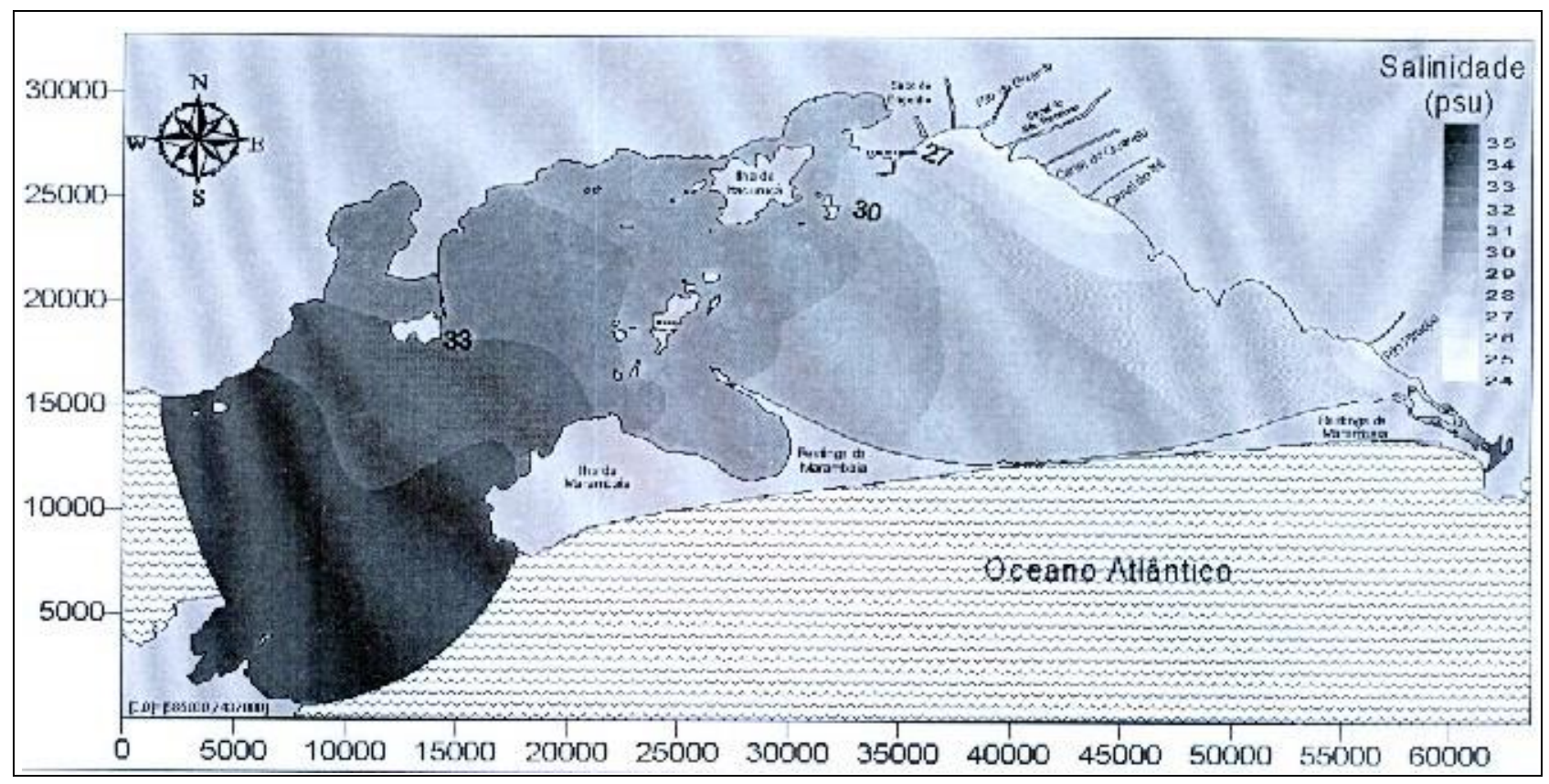

Figura 13. Distribuição de salinidade simulada com o FIST-ADE próxima ao instante de preamar (15:00 h de 18/04/96), em situação de maré de sizígia, considerando maré astronômica e maré meteorológica. Verifica-se uma significativa variação nos valores de salinidade principalmente na região próxima à fronteira aberta (comparar com Figura 12). 
siderados iguais a 24 PSU (Practical Salinity Unit, correspondendo ao valor da salinidade em \%, g/l ou $\mathrm{kg} / \mathrm{m}^{3}$ ), para todos os rios. Os demais parâmetros de calibração usados para simular o transporte de sal na Baía podem ser observados na Tabela 4.
Nas Figuras 12 a 17 são mostradas as distribuições de salinidade na Baía de Sepetiba. Notese que a Baía é quase homogênea, uma vez que os gradientes de salinidade concentram-se em uma faixa estreita, próxima aos rios. As fontes de água do-

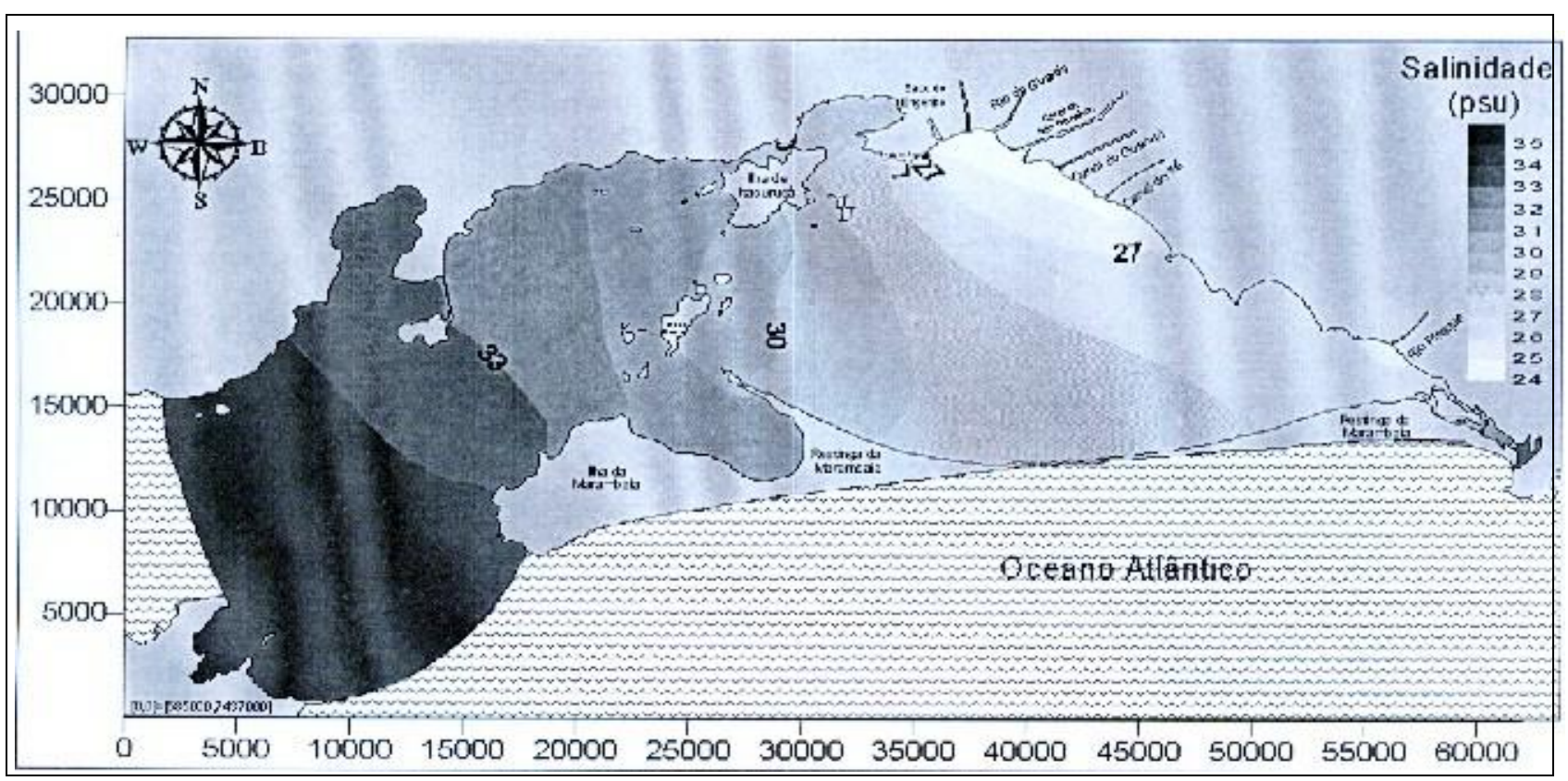

Figura 14. Distribuição de salinidade simulada com o FIST-ADE próxima ao instante de baixa-mar (06:00 h de 25/04/96), em situação de maré de quadratura, considerando maré astronômica e maré meteorológica.

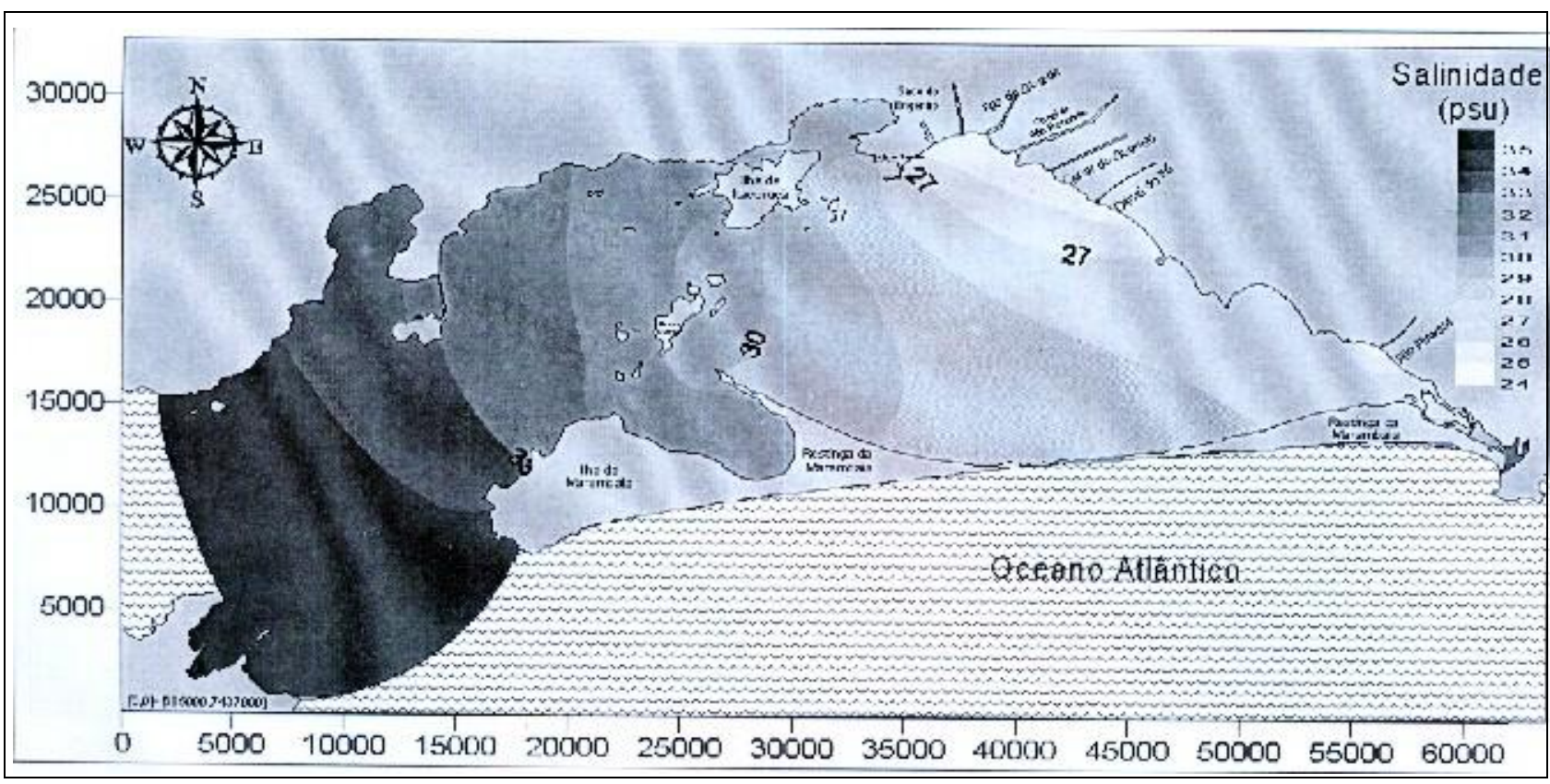

Figura 15. Distribuição de salinidade simulada com o FIST-ADE próxima ao instante de preamar (15:00 h de 25/04/96), em situação de maré de quadratura, considerando maré astronômica e maré meteorológica. Verifica-se uma pequena variação nos valores de salinidade ao longo de toda a Baía (comparar com Figura 14). 
ce vêm dos seis rios considerados na circulação hidrodinâmica. Nessa simulação, a salinidade no contorno aberto foi prescrita como 35 PSU e usada como valor de referência. Estes resultados estão qualitativamente concordantes com os valores encontrados pela FEEMA/GTZ (Fundação Estadual de Engenharia do Meio Ambiente e Projeto de Cooperação Técnica Brasil-Alemanha) durante este pe-

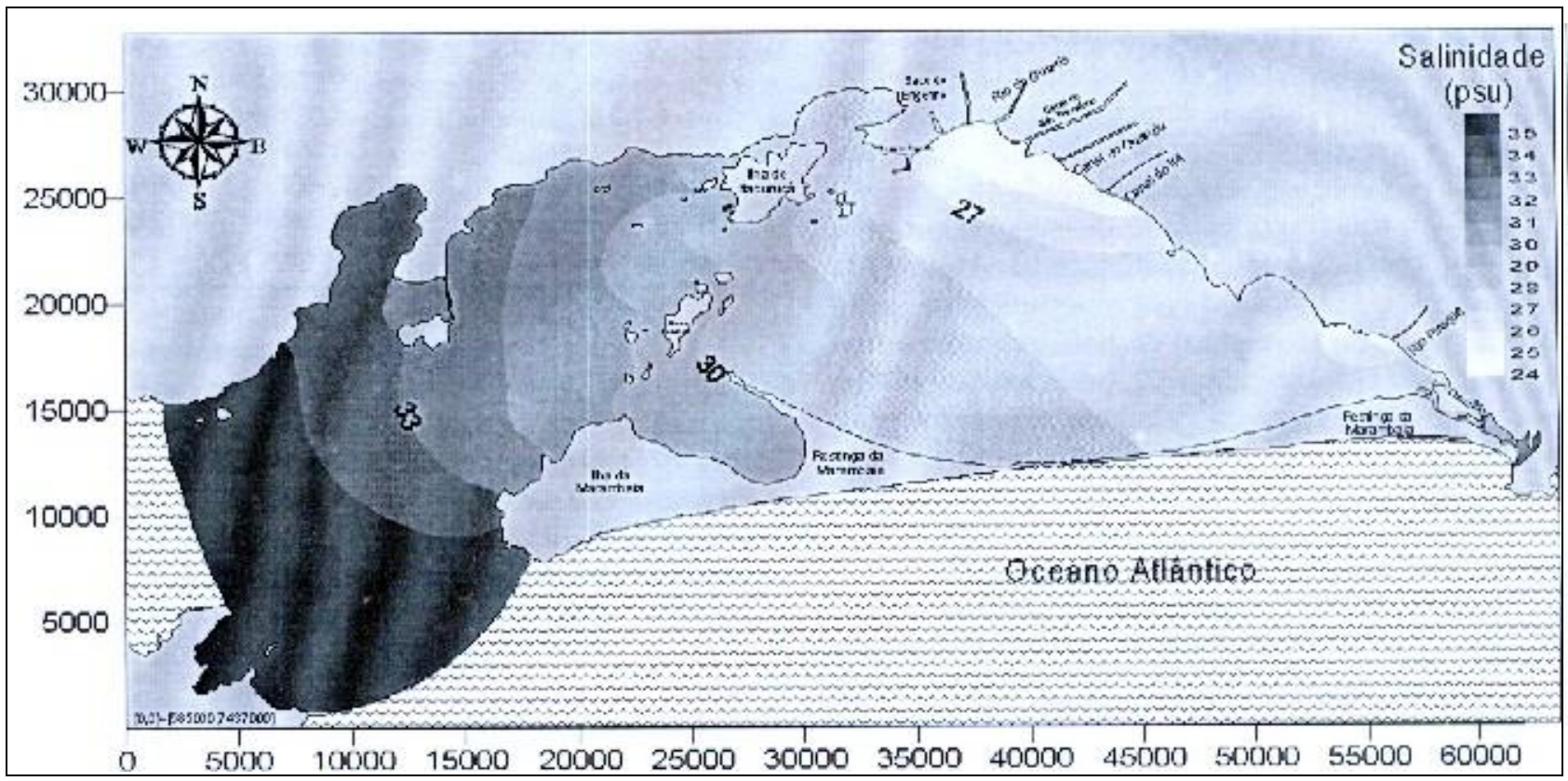

Figura 16. Distribuição de salinidade simulada com o FIST-ADE próxima ao instante de baixa-mar (09:00 h de 03/05/96), em situação de maré de sizígia. Comparando com a Figura 12, os valores de salinidade não são intensificados quando não há entrada de frente fria.

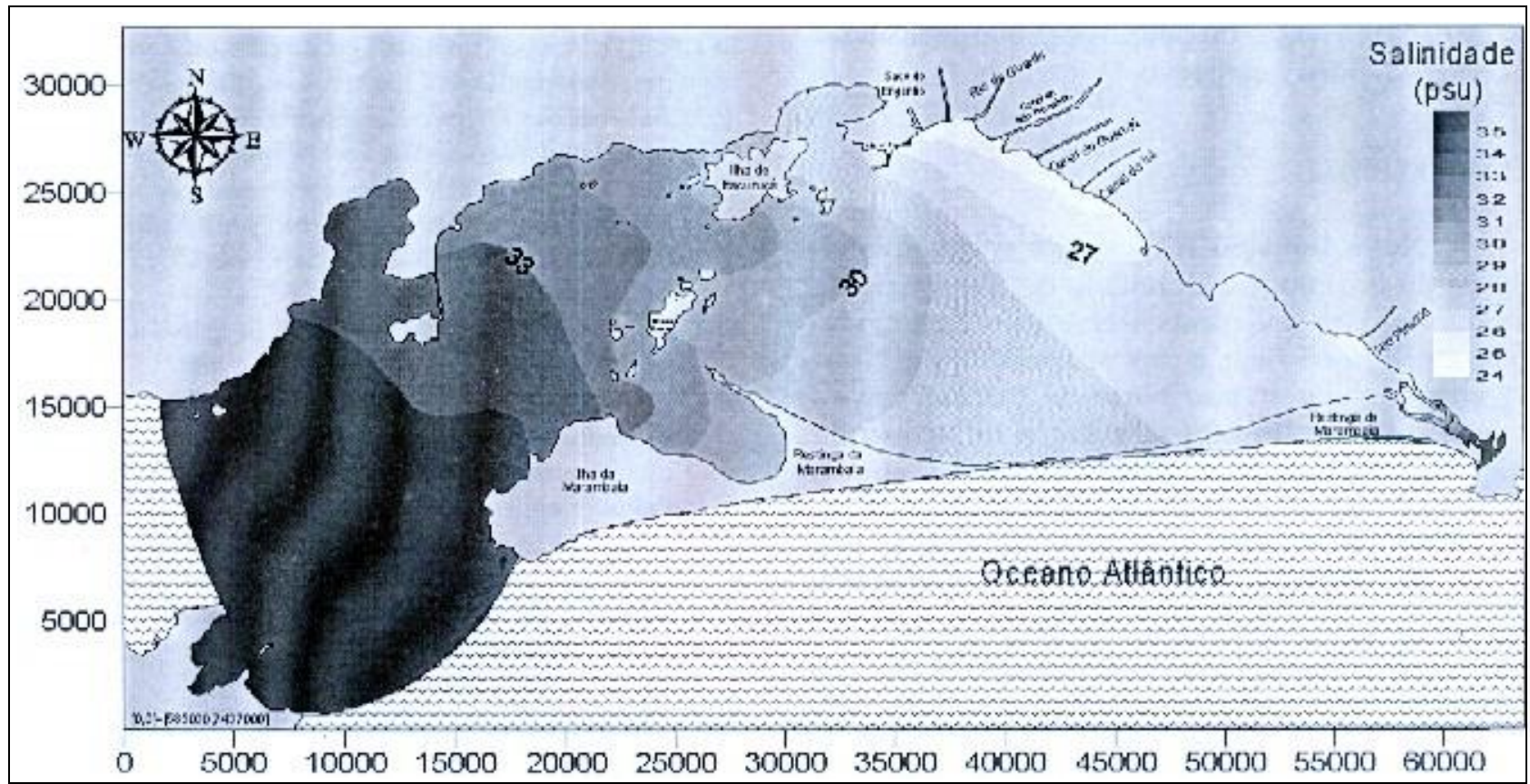

Figura 17. Distribuição de salinidade simulada com o FIST-ADE próxima ao instante de preamar (15:00 h de 03/05/96), em situação de maré de sizígia. Comparando com a Figura 13, os valores de salinidade não são intensificados quando não há entrada de frente fria. 
ríodo e parecem suficientemente consistentes, considerando que o modelo calcula um valor de salinidade médio na vertical.

Observa-se ainda que não há grandes variações da distribuição de salinidade em situações de maré de quadratura e sizígia, sem a entrada de frente fria (Figuras 14 a 17). Pode-se afirmar que a salinidade pouco varia durante um ciclo de maré de quadratura, já que os gradientes de salinidade permanecem relativamente estáveis durante todo o ciclo.

Em situações de maré de sizígia, considerando a entrada de frente fria (Figura 12 e Figura 13), os gradientes de salinidade são intensificados, principalmente na região central da Baía, próxima à Ilha de Itacuruçá. Esta intensificação pode ser atribuída à sobre-elevação provocada pela maré meteorológica, que facilita a entrada de sal na Baía. Na parte mais interna da Baía, as misturas entre as águas mais doces, provenientes dos rios, e as águas oceânicas é menor, com uma tendência de estabilização.

Pode-se ainda concluir que a Baía de Sepetiba é um corpo d'água com águas salobras e salinas, já que a salinidade dentro da Baía varia entre 24 PSU e 35 PSU. Vale lembrar que esta aplicação é apenas ilustrativa das potencialidades do FIST-ADE na modelagem ambiental, sendo, portanto, conclusiva apenas de modo qualitativo, no que diz respeito à distribuição de salinidade na Baía de Sepetiba. Os resultados aqui apresentados precisam ser calibrados e validados com dados de campo para que possam ser usados de modo quantitativo.

\section{CONCLUSÃO}

Neste trabalho é mostrado o desenvolvimento de um modelo Eureliano de transporte de substâncias passivas não-conservativas. Este modelo se mostrou eficiente e preciso, podendo ser usado para simular o transporte de sal em corpos d'água costeiros. Mostra-se ainda a influência da interface dissipativa na solução numérica, com o controle das oscilações numéricas e sem provocar qualquer amortecimento apreciável na solução.

A validação do código foi feita através da comparação com resultados analíticos conhecidos, levando em consideração diferentes parâmetros de simulação. Nos casos mostrados, o modelo demonstrou um bom desempenho no controle das oscilações numéricas, quando da utilização de condições adversas de simulação. Em condições favoráveis, o resultado numérico é uma excelente aproximação da solução analítica.
Tabela 4. Parâmetros de entrada do modelo de transporte de sal.

\begin{tabular}{cc}
\hline Parâmetros & Valor \\
\hline$\alpha$ & 1.0 \\
$\beta$ & 1.0 \\
$\Lambda_{\mathrm{x}}$ & 2.0 \\
$\Lambda_{\mathrm{y}}$ & 2.0 \\
$\Lambda_{\mathrm{t}}$ & 2.0 \\
$\mathrm{C}^{*}$ & $35.0 \mathrm{psu}$ \\
$\tau$ & $3600.0 \mathrm{~s}$. \\
\hline
\end{tabular}

Na aplicação do transporte de sal na Baía de Sepetiba, o modelo mostrou-se capaz de simular o transporte de escalares distribuídos na área da Baía, obtendo resultados consistentes com os valores observados e mostrando que o sistema de modelos é adequado para os objetivos do estudo. Vale lembrar que esta aplicação representa um primeiro estágio no longo processo de calibração dos modelos de circulação e de transporte na Baía de Sepetiba.

\section{REFERÊNCIAS}

ALDAMA, A. (1985). Theory and applications of two - and three-scale filtering approaches for turbulent flow simulation. Ph.D. Thesis, Dept. Civil Engineering, Massachusetts Institute of Technology.

BEDFORD, K. (1994). Diffusion, dispersion and sub-grid parameterization. Chapter 4 in "Coastal, Estuarial and Harbour Engineers' Reference Book", Ed. by M. B. Abbot and W. A. Price, E\&FN Spon.

CUNHA, C. L. N. (2000). Desenvolvimento de um modelo hidrodinâmico tridimensional sem aproximação hidrostática. Tese D. Sc., Engenharia Civil, COPPE/UFRJ, Brasil.

FISCHER, H. B. (Ed.) (1979). Mixing in Inland and coastal waters. Academic Press Inc., New York.

HSU, M-H.; KUO, A. Y.; KUO, J-T \& LIU, W-C. (1999). Procedure to calibrate and verify numerical models of estuarine hydrodynamics. Journal of Hydraulic Engineering, vol. 125, $\mathrm{n}^{\circ} 2$.

JORGE, A. L. F. A. (1999). Uso de GMRES para redução do tempo de simulação hidrodinâmica pelo método dos elementos de contorno e pelo método dos elementos finitos. Seminário de Doutorado, Engenharia Civil COPPE/UFRJ. Rio de Janeiro, RJ, Brasil.

NGUYEN, K. D. \& OUAHSINE, A. (1997). 2D numerical study on tidal circulation in strait of dover. Journal of Waterway, Port, Coastal, and Ocean Engineering, vol. $123, \mathrm{n}^{\circ} 1$

OLIVEIRA, A.; FORTUNATO, A. B. \& BATISTS, A. M. (2000). Mass balance in Eulerian-Lagrangins 
transport simulations in estuaries. Journal of Hydraulic Engineering, vol. 126, nº8.

ROSMAN, P. C. C. (1987). Modeling shallow water bodies via filtering techniques. Ph.D. Thesis, Dept. of Civil Engineering, Massachusetts Institute of Technology.

ROSMAN, P. C. C. (2000). Referência técnica do SISBAHIA - Sistema Base de Hidrodinâmica Ambiental. Programa COPPE: Engenharia Oceânica, Área de Engenharia Costeira e Oceanográfica, Rio de Janeiro, Brasil.

ROSMAN, P. C. C. \& GOBBI, E. F. (1990). A self-adjusting subgrid "turbulence" model for shallow water flow. XI Ibero American Congress on Computational Methods in Engineering, 10/90. Rio de Janeiro.

SAAD, Y. (1995). Iterative methods for sparse linear systems. PWS Publishing Company, EUA.

VINZON, S. B. (1991). Modelação de transporte de substância passivas em corpos de água rasos. Tese M. Sc., Engenharia Oceânica, COPPE/UFRJ, Brasil.

ZIENKIEWICZ, O. C. \& MORGAN, K. (1982). Finite elements and approximation. John Wiley\&Sons, New York.

\section{Two Dimensional Modeling of Scalar Transport}

\section{ABSTRACT}

This paper presents the development of a twodimensional transport model that simulates the transport of substances, i. e., contaminants that are well mixed in the water column, applied as passive and nonconservative scalars. The model employs a depthaveraged advective diffusive transport model; the finite element method is employed in the spatial discretization and a finite difference scheme is adopted in order to perform the time-domain analysis. Turbulent stresses are taken into account by means of techniques similar to those employed in Large Eddy Simulation (LES).

Model validation is performed by comparing its results with analytical and experimental results, considering different sources. The model is also employed to simulate salt transport in Sepetiba Bay, simply to illustrate practical applications in estuaries with complex geometric and bottom topography.

Key Words: two dimensional modeling; scalar transport. 